\title{
Spatial specificity of the functional MRI blood oxygenation response relative to neuronal activity
}

Denis Chaimow ${ }^{1}$, Essa Yacoub ${ }^{1}$, Kâmil Uğurbil ${ }^{1}$, Amir Shmuel ${ }^{1,2}$

${ }^{1}$ Centre for Magnetic Resonance Research

University of Minnesota, Minneapolis, USA

${ }^{2}$ McConnel Brain Imaging Centre, Montreal Neurological Institute

Departments of Neurology, Neurosurgery, Physiology and Biomedical Engineering

McGill University, Montreal, QC, Canada

Keywords

High-resolution functional MRI, Point-spread function, Blood oxygenation level dependent, BOLD, Gradient-Echo functional MRI, Spin-Echo functional MRI, Cortical columns, Ocular dominance map, Markov chain Monte Carlo

Acknowledgements

This work was supported by grants from the Natural Sciences and Engineering Research Council of Canada (AS, NSERC Discovery grants RGPIN 375457-09 and RGPIN-2015-05103). 


\begin{abstract}
Previous attempts at characterizing the spatial specificity of the blood oxygenation level dependent functional MRI (BOLD fMRI) response by estimating its point-spread function (PSF) have conventionally relied on spatial representations of visual stimuli in area V1. Consequently, their estimates were confounded by the width and scatter of receptive fields of V1 neurons. Here, we circumvent these limits by instead using the inherent cortical spatial organization of ocular dominance columns (ODCs) to determine the PSF for both Gradient Echo (GE) and Spin Echo (SE) BOLD imaging at 7 Tesla. By applying Markov Chain Monte Carlo sampling on a probabilistic generative model of imaging ODCs, we quantified the PSFs that best predict the spatial structure and magnitude of differential ODCs' responses. Prior distributions for the ODC model parameters were determined by analyzing published data of cytochrome oxidase patterns from post-mortem histology of human V1 and of neurophysiological ocular dominance indices. The most probable PSF full-widths at halfmaximum were $0.82 \mathrm{~mm}$ (SE) and $1.02 \mathrm{~mm}$ (GE). Our results provide a quantitative basis for the spatial specificity of BOLD fMRI at ultra-high fields, which can be used for planning and interpretation of high-resolution differential fMRI of fine-scale cortical organizations.
\end{abstract}

\title{
Introduction
}

Functional magnetic resonance imaging (fMRI) of the human brain is increasingly being used to investigate fine-scale structures such as cortical columns (Cheng et al., 2001; De Martino et al., 2015; Goodyear and Menon, 2001; Menon et al., 1997; Shmuel et al., 2010; Yacoub et al., 2008; 2007; Zimmermann et al., 2011). To optimally plan high-resolution fMRI studies and to correctly interpret their results it is necessary to know the inherent limits of the fMRI spatial specificity relative to the sites where changes in neuronal activity occur.

The most commonly used fMRI approach relies on gradient echo (GE) blood oxygenation level dependent (BOLD) contrast (Bandettini et al., 1992; Kwong et al., 1992; Ogawa et al., 1990; 1992). GE BOLD is sensitive to the intraand extravascular effects of activation-induced changes in the deoxyhemoglobin content of blood. At standard magnetic field strengths (1.5 T, $3 \mathrm{~T}$ ) the signal is dominated by contributions from larger blood vessels. At higher magnetic field strengths the strong intravascular component of these large blood vessels decreases, while the extravascular signal changes around capillaries and smaller vessels increase (Uludağ et al., 2009; Yacoub et al., 2001). Additional weighting towards the microvasculature can be achieved by using spin echo (SE) BOLD imaging, which suppresses extravascular signal contributions from larger blood vessels (Uludağ et al., 2009; Yacoub et al., 2003).

The first study to quantify the spatial specificity of the BOLD response (Engel et al., 1997) used an elegant phase-encoding paradigm that induced traveling waves of retinotopic neural activity in the primary visual cortex (V1). Assuming a shift-invariant linear response, Engel et al. (1997) estimated the point-spread function (PSF), which represents the spatial response that would 
be elicited by a small point stimulus. They found the full-width at halfmaximum (FWHM) of the GE BOLD PSF to be $3.5 \mathrm{~mm}$ at $1.5 \mathrm{~T}$. Similar values (3.9 $\mathrm{mm}$ for GE BOLD and $3.4 \mathrm{~mm}$ for SE BOLD) have been reported at $3 \mathrm{~T}$ (Parkes et al., 2005) using a paradigm similar to that used in Engel et al. (1997). To estimate the GE BOLD PSF at 7 T, we previously measured the spatiotemporal spread of the fMRI response in grey matter regions around the V1 representation of edges of visual stimuli (Shmuel et al., 2007). To reduce contributions from macroscopic veins, we excluded voxels that showed vessel-like response features. The mean measured and estimated FWHMs were $2.34 \pm$ $0.20 \mathrm{~mm}$ and $<2 \mathrm{~mm}$, respectively. The spatial specificity of SE BOLD fMRI at ultra-high magnetic fields has not yet been quantified.

All previous attempts at characterizing the spatial specificity of the BOLD fMRI response (Engel et al., 1997; Parkes et al., 2005; Shmuel et al., 2007) relied on an implicit assumption that neuronal responses to small visual stimuli are point-like. However, to estimate the spatial specificity of the BOLD response, these studies have conventionally relied on spatial representations of visual stimuli in area V1. Unlike the implicit assumption of point-like responses, the receptive fields of neurons in V1 have non-zero spatial extents (Hubel and Wiesel, 1968). In addition, electrode measurements in macaque V1, oriented orthogonally relative to the surface of cortex have demonstrated substantial scatter in the center of receptive fields (Hubel and Wiesel, 1974). Therefore, the pattern of neural activity parallel to the cortical surface is a blurred representation of the visual stimulus. This implies that receptive field size and scatter pose a lower limit on any BOLD fMRI PSF width that is estimated using spatial representations of visual stimuli in V1. Consequently, the previously computed estimates of the spatial specificity of the fMRI response were confounded by the width and scatter of receptive fields of V1 neurons. Such estimates are limited in that they solely measure the capacity of the BOLD response to resolve retinotopic representations; they do not measure its ability to resolve more fine-grained neural activity. Yet only this latter resolvability matters for functional imaging at the spatial scale of cortical columns.

Here, we estimate and compare the PSF widths of GE and SE BOLD imaging at $7 \mathrm{~T}$ using a novel approach. We circumvent the limits posed by the retinotopic representation of visual stimuli by instead using the inherent cortical spatial organization of ocular dominance columns (ODCs). To this end, we fit a model of ODCs imaging (Chaimow et al., 2011) to ODCs responses acquired at $7 \mathrm{~T}$ (Yacoub et al., 2007). We quantify the width of the PSF that best predicts the spatial structure and magnitude of differential ODC responses. Since we do not have access to the underlying anatomical ODC patterns and neurophysiological responses, we use a probabilistic modeling approach. We constrain the model ODC parameters by estimating features of real ODC patterns taken from post-mortem cytochrome oxidase (CO) maps of human ODCs (Adams et al., 2007) and neurophysiological response distributions in primates (Berens et al., 2008; Hubel and Wiesel, 1968). We then fit our model by Markov Chain Monte Carlo (MCMC) sampling. Our results provide a quantitative basis for the spatial specificity of differential BOLD fMRI at ultra-high fields. 


\section{Methods}

\section{Overview}

We developed a probabilistic generative model of imaging ODCs in order to estimate widths of GE and SE BOLD PSFs that would best explain our previously obtained fMRI data of differential ODC maps (Yacoub et al., 2007).

The measured fMRI maps consisted of voxel estimates of the difference in BOLD responses to left and right eye stimulation. We modeled these responses as the sum of predictions from an ODC imaging model and measurement noise. The predictions from the ODC imaging model were completely determined by a set of parameters. Accounting for the effect of the measurement noise allowed us to first express the probability of observing the measured fMRI maps as a function of model parameters. In a second stage, we derived the posterior probability of the model parameters given the observed data and the prior probability of parameters.

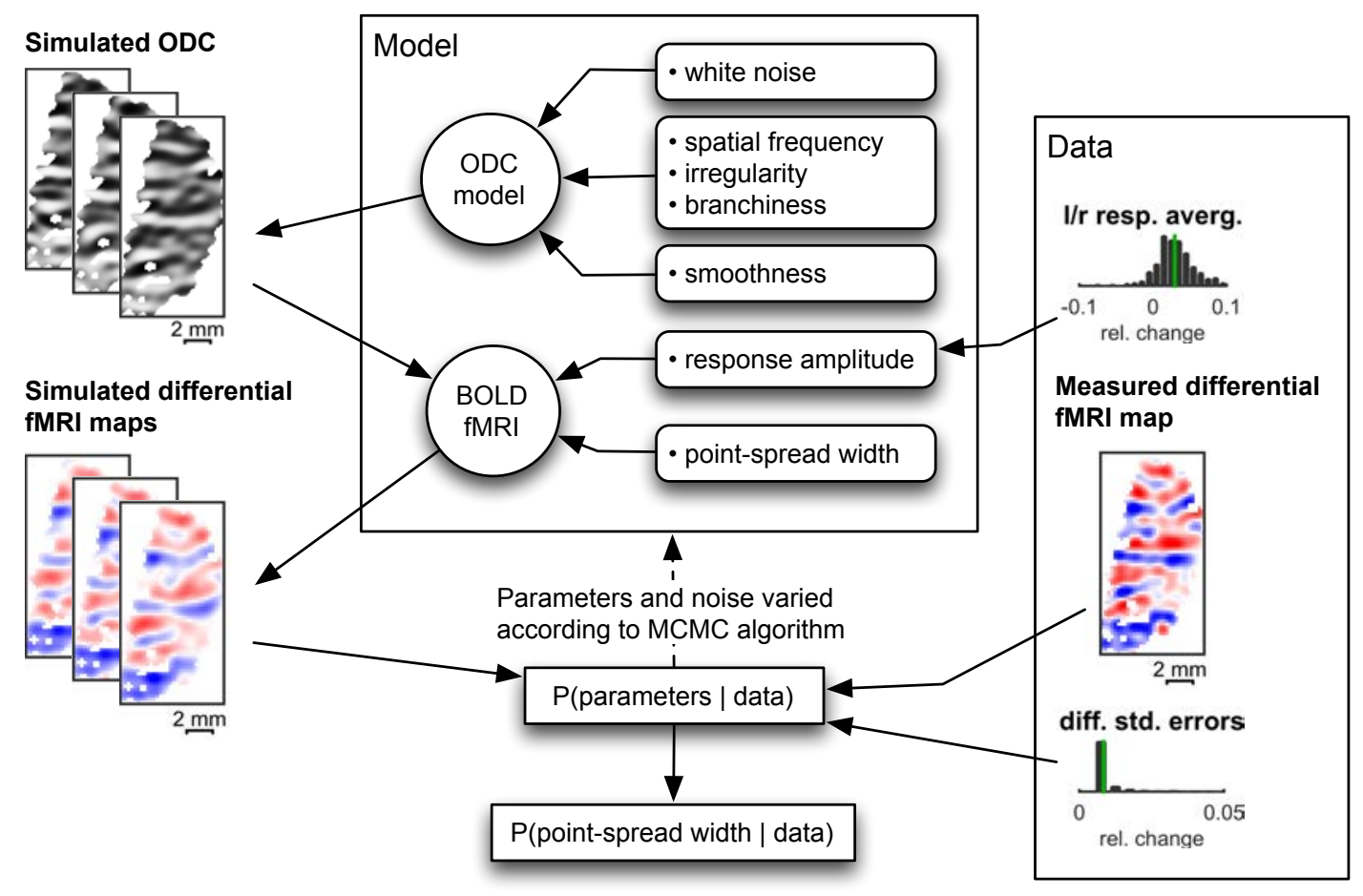

Fig. 1 Overview of Markov Chain Monte Carlo fitting. The model was fitted to the fMRI data using Markov Chain Monte Carlo (MCMC) sampling. For an arbitrary given set of parameters, the model generated a differential fMRI map (left). This map was compared to the measured fMRI map (right) and the likelihood of parameters given the data was calculated. The MCMC algorithm uses this likelihood together with parameter priors to further traverse the parameter space. After sufficiently many iterations the resulting parameter samples are distributed according to their joint posterior probability distribution.

\section{Model of imaging ODCs}

We implemented a model of imaging ODCs (Chaimow et al., 2011; see Fig. 1 for an overview; see Appendix A in the Suppl. Material for the detailed equations). The first component of the model, i.e. the modeling of realistic ODCs, followed (Rojer and Schwartz, 1990). It consisted of band-pass filtering of spatial white 
noise using an anisotropic filter. The filtering was followed by applying a sigmoidal point-wise non-linearity, which controlled the smoothness of transitions between left and right eye preference regions.

The spatial BOLD response was modeled as a convolution of the ODCs pattern with a Gaussian PSF. We modeled separate BOLD responses for the GE and SE maps. However, as these maps were obtained from the exact same region in area V1 in each subject, the model constituted one ODC map underlying both the GE and SE responses. MRI k-space sampling was modeled by restricting the spatial frequency space to its central part in accordance with the modeled field of view, sampling matrix and voxel size (Haacke et al., 1999).

All model parameters are listed in Table 1. Three parameters controlled general ODC properties: the main (peak) spatial frequency $\rho$, the degree of irregularity $\delta$ and the branchiness $\epsilon$. The spatial white noise served as a highdimensional parameter determining the specific manifestation of the ODC pattern. A parameter $\omega$ controlled the smoothness of transitions between regions showing left and right eye preference. The PSFs of the BOLD responses were parameterized by amplitudes $\beta_{G E}$ and $\beta_{S E}$ and by their FWHM $f w h m_{G E}$ and $f w h m_{S E}$ (the parameters of interest).

Relative to our previously published model, we made 2 slight modifications. First, to simplify derivation of the gradient we subsequently used for implementing the MCMC sampling, we modified the formulation of the bandpass filtering kernel (see Appendix A). Second, due to consideration of step size determined by the MCMC algorithm, we defined and used a smoothness parameter $\omega$ instead of using its inverse, the sharpness parameter a which we used previously.

\section{Model implementation}

The model was implemented in MATLAB (The MathWorks Inc., Natick, MA, USA). All model computations were carried out on a Cartesian grid of $0.125 \times$ $0.125 \mathrm{~mm}^{2}$ resolution. Spatial filtering used for the ODC and BOLD PSF modeling was carried out in the frequency domain using discrete Fourier transforms. The discrete Fourier transform assumes signals to be periodic, thereby forcing opposite edges of the grid to be continuous. In order to minimize this effect on modeling, the simulated area was extended relative to the data by doubling the length of each dimension.

\section{Prior estimation}

Table 1 presents an overview of employed priors for all parameters. The spatial Gaussian white noise had an independent multivariate normal distribution with a standard deviation of 1 as its prior. Priors for the ODC model parameters were estimated from anatomical and neurophysiological data as described in the following sections. 
Table 1 Model Parameters. Free parameters are probabilistic parameters. We used MCMC to sample from their joint distribution. Fixed parameters were estimated directly from the data and were held constant during MCMC sampling.

\begin{tabular}{|c|c|c|c|}
\hline $\begin{array}{l}\text { Free } \\
\text { parameters }\end{array}$ & Description & Prior & MCMC starting value \\
\hline$n_{i, j}$ & $\begin{array}{l}\text { Spatial white noise to be } \\
\text { filtered, determines } \\
\text { specific ODC pattern }\end{array}$ & $\begin{array}{l}\text { Multivariate standard } \\
\text { normal distribution }\end{array}$ & $\begin{array}{l}\text { Up-sampled differential } \\
\text { fMRI map, normalized to } \\
\text { have a standard deviation } \\
\text { of } 1\end{array}$ \\
\hline$\rho$ & $\begin{array}{l}\text { Main spatial frequency of } \\
\text { ODC }\end{array}$ & $\begin{array}{l}\text { Limited normal } \\
\text { distribution, parameters } \\
\text { estimated from } \\
\text { anatomical data }\end{array}$ & $\begin{array}{l}\text { Set to mean of estimates } \\
\text { from anatomical data }\end{array}$ \\
\hline$\delta$ & Irregularity & $\begin{array}{l}\text { Normal distribution, } \\
\text { parameters estimated } \\
\text { from anatomical data }\end{array}$ & $\begin{array}{l}\text { Set to mean of estimates } \\
\text { from anatomical data }\end{array}$ \\
\hline$\epsilon$ & Branchiness & $\begin{array}{l}\text { Normal distribution, } \\
\text { parameters estimated } \\
\text { from anatomical data }\end{array}$ & $\begin{array}{l}\text { Set to mean of estimates } \\
\text { from anatomical data }\end{array}$ \\
\hline$\omega$ & $\begin{array}{l}\text { Smoothness of } \\
\text { transitions }\end{array}$ & $\begin{array}{l}\text { Uniform between } 0.3 \text { and } \\
2 \text { based on } \\
\text { neurophysiology data }\end{array}$ & Set to mean of limits \\
\hline $\boldsymbol{\theta}$ & Orientation of ODC & Flat & $\begin{array}{l}\pi / 2 \text { (corresponds to ODC } \\
\text { bands parallel to medio- } \\
\text { lateral direction) }\end{array}$ \\
\hline$f w h m_{G E}$ & $\begin{array}{l}\text { GE point-spread-function } \\
\text { width (full width at half } \\
\text { maximum) }\end{array}$ & Flat & $2 \mathrm{~mm}$ \\
\hline$f_{w h m_{S E}}$ & $\begin{array}{l}\text { SE point-spread-function } \\
\text { width (full width at half } \\
\text { maximum) }\end{array}$ & Flat & $2 \mathrm{~mm}$ \\
\hline $\begin{array}{l}\text { Fixed } \\
\text { parameters }\end{array}$ & Description & Estimation & \\
\hline$\beta_{G E}$ & GE response amplitude & \multicolumn{2}{|c|}{$\begin{array}{l}\text { Twice the median across voxels of the average between } \\
\text { GE responses to left and right eye stimulation }\end{array}$} \\
\hline $\boldsymbol{\beta}_{S E}$ & SE response amplitude & \multicolumn{2}{|c|}{$\begin{array}{l}\text { Twice the median across voxels of the average between } \\
\text { SE responses to left and right eye stimulation }\end{array}$} \\
\hline$\widehat{\sigma}_{G E}^{2}$ & $\begin{array}{l}\text { GE measurement noise } \\
\text { variance }\end{array}$ & \multicolumn{2}{|c|}{$\begin{array}{l}\text { Mean across voxels of estimation variance of differential } \\
\text { GE response }\end{array}$} \\
\hline$\widehat{\sigma}^{2}{ }_{S E}$ & $\begin{array}{l}\text { SE measurement noise } \\
\text { variance }\end{array}$ & \multicolumn{2}{|c|}{$\begin{array}{l}\text { Mean across voxels of estimation variance of differential } \\
\text { SE response }\end{array}$} \\
\hline
\end{tabular}

Estimation of priors from cytochrome oxidase data

Four single hemisphere images of complete patterns of ODCs in the human brain taken from Adams et al. (2007) were reanalyzed. These images were originally obtained by postmortem staining for CO activity in human subjects who had lost one eye. The goal of this analysis was to find model parameters that gave rise to modeled ODC maps whose spatial power spectra most closely resemble those of real human ODCs (Fig. 2). 


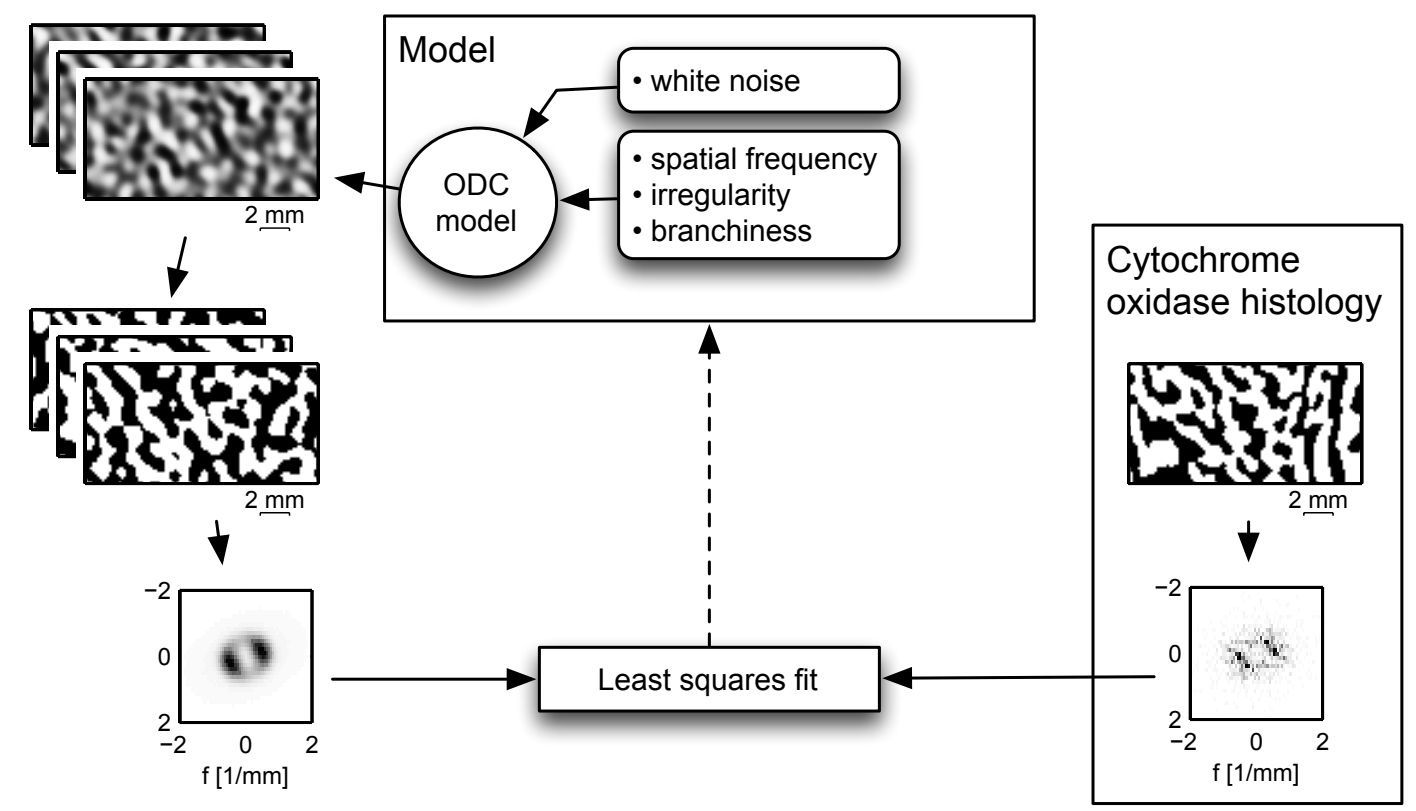

Fig. 2 Overview of cytochrome oxidase fitting. In order to constrain the parameters of the ODC model (main spatial frequency, irregularity and branchiness), cytochrome oxidase (CO) maps of human ODCs (Adams et al., 2007) were analyzed. Model parameters were optimized so that the spatial power spectra of binary ODC maps generated by the model (left) resembled those of the CO maps (right).

Two rectangular regions from each image, which corresponded in cortical location and extent to our fMRI ODC maps, were selected. To this end, V1 boundaries and the representation of the fovea were delineated. The eccentricity for each point in V1 was computed from the cortical distance to the fovea $d_{\text {fovea }}$ as $0.75+\exp \left(\frac{d_{\text {fovea }}}{17.3}\right)$ (Horton and Hoyt, 1991). For every point in the map, the two locations on the upper and lower V1 boundary (representing the vertical meridians) with eccentricity equal to that of the considered point were identified. The angular distances (along points with the same eccentricity) from the point under consideration to each of those two points on the boundary were calculated. The horizontal meridian was defined as the set of all V1 points for which those two distances were equal (green line in Fig. 3A).

The two regions to be selected, corresponding to the upper and lower banks of the calcarine sulcus, were then defined using the following criteria. First, the spatial extent was set to $15.7 \mathrm{~mm} \times 8 \mathrm{~mm}$, so that the area was equal to the mean area of our fMRI regions of interest (ROI) and the aspect ratio was equal to the mean aspect ratio of our fMRI ROIs. Second, ROIs had to be $5 \mathrm{~mm}$ away from the horizontal meridian and centered within an eccentricity range of $3^{\circ}$ to $10^{\circ}$, corresponding to the expected location of the flat regions of the calcarine sulcus (Cheng et al., 2001).

The pattern of the CO map was binarized, in order to obtain the pattern of absolute ocular dominance (i.e. left or right eye preference). Then, for each map, we fitted the parameters of the ODC part of the model such that the spatial power spectra of the simulated binarized ODC maps were similar to those of the CO maps of ODCs (Fig. 3B, measured ODC; Fig. 3C, simulated ODC). For a model that consists of the filtering of spatial white noise only (i.e. with- 
out a sigmoidal point-wise non-linearity or binarization), the frequency spectrum of the output is expected to resemble the filter shape. Here, the spatial frequency spectra of the binarized CO maps were used to obtain first estimates of the ODC filter parameters. To that end, spectra were resampled to polar coordinates and their radial and angular averages were computed. Model equations for the radial and angular filter components (see Appendix A) were fitted to these averages using the MATLAB Curve Fitting Toolbox, enabling the extraction of parameter estimates $(\rho, \delta, \theta, \epsilon)$. Random binary ODC patterns (400 in each step) were simulated using these estimates as initial values. Their power spectra were averaged, and the sum of squared differences between the data spectrum and the average simulated spectrum was computed. An optimization algorithm in MATLAB (fminsearch; Lagarias et al., 1998) was used to find parameters that minimized this sum of squared differences.

A

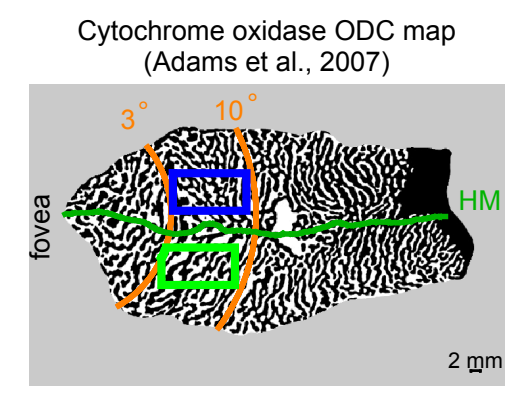

B
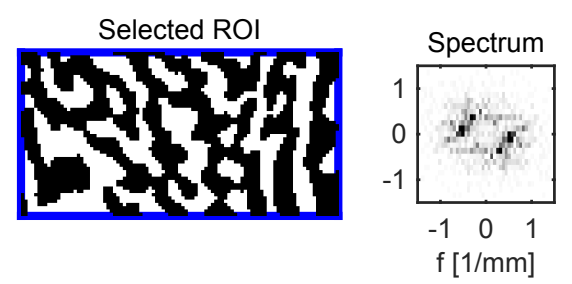

C

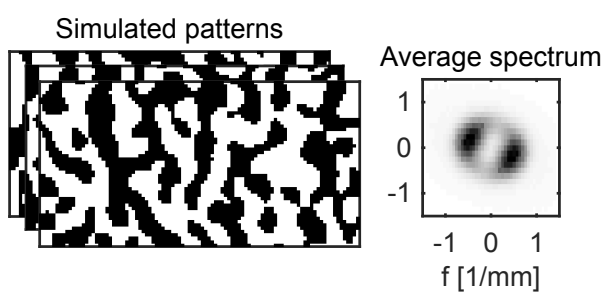

D

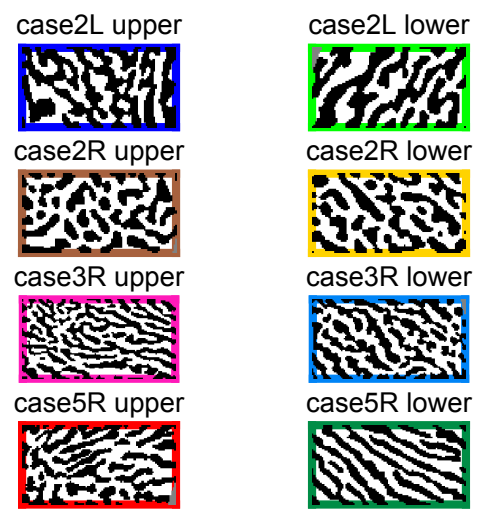

E
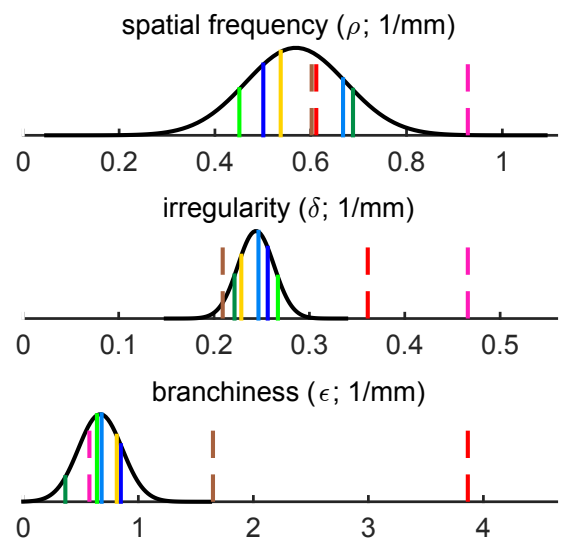

Fig. 3 Results of cytochrome oxidase ODC map analysis. A Cytochrome oxidase ODC maps from human V1 were imported from Adams et al. (2007). Regions of interest (ROIs) were selected to be comparable in size and location to our fMRI data. B The ODC pattern from the selected upper bank region (left) is shown next to its spatial power spectrum (right). C Model parameters were optimized to produce simulated patterns (left) whose average spectrum (right) was comparable to the spectrum of the data in B. D The patterns from the upper and lower bank regions of all four cases are shown with color-coded surrounds. E The estimated values of model parameters from all patterns are shown as vertical lines using the same colors as in D. Dashed lines indicate values from patterns that were classified as outliers in the distribution of spatial frequency, irregularity, or branchiness. The mean and standard deviation of the remaining values were used to define Gaussian distributions (black) to be used as priors for fitting the model to the fMRI data. 
Three maps and their fitted parameters showed outlier features. For example, 'case5R upper' (Fig 3D, red surround) showed very thin bands in one region (upper left) immediately adjacent to a region of thicker bands (bottom right), resulting in outlier estimates of irregularity and branchiness (red lines in Fig. 3E, middle and bottom panels). Such abrupt changes may have resulted from the processing of anatomical specimens or from the presence of curved boundaries between locally flat regions.

In order to avoid atypical parameters estimates, parameter values whose absolute deviation from the median exceeded 3.7 times the median absolute deviation (corresponding to 2.5 standard deviations of a normal distribution; Leys et al., 2013) were marked as outliers. Maps in which at least one parameter was marked as an outlier were excluded from further analysis. This procedure resulted in the exclusion of three maps (cases $2 \mathrm{R}, 3 \mathrm{R}$ and $5 \mathrm{R}$ upper) from further analysis.

For each parameter, model priors were defined as normal distributions with means and standard deviations equal to the sample means and standard deviations of parameter values from the remaining maps. In order to further discourage extreme parameter values, we set the prior for $\rho$ outside two standard deviations from the mean to zero.

Estimation of a prior for the smoothness parameter $\omega$

A prior for the smoothness parameter $\omega$ was constructed on the basis of ocular dominance indices (ODIs) as reported in the neurophysiological literature. We assumed that ODI distributions in humans are similar to those in the macaque.

ODIs are defined as $O D I=\frac{y_{\text {left }}-y_{\text {right }}}{y_{\text {left }}+y_{\text {right }}}$, where $y_{\text {left }}$ and $y_{\text {right }}$ denote the response values to stimuli presented to the left and right eye respectively (e.g. Berens et al., 2008). ODIs were calculated from differential ODC maps generated by our model and fit to ODI distributions from the literature (Berens et al., 2008; Hubel and Wiesel, 1968). $\omega$ was allowed to vary while all other parameters were fixed as the mean of their anatomical data estimates. The value of $\omega$ that resulted in the smallest Kullback-Leibler divergence between the modeled and the target distribution was selected.

In order to fit modeled ODI distributions to the seven-class classification of Hubel and Wiesel (1968), classes 1 and 7 were collapsed into an exclusively responding class (left or right eye). Classes 2, 3, 5 and 6 were collapsed into an intermediately responding class, with class 4 responding indifferently. Modeled ODI distributions were also transformed into these three classes. The range of absolute ODIs that were assigned to the exclusively responding class and to the indifferently responding class were defined by a class width parameter. The values of $\omega$ and of the width parameter that together resulted in the smallest Kullback-Leibler divergence between the modeled and the target distribution were selected.

\section{fMRI Data acquisition}

7 T BOLD fMRI data from Yacoub et al. (2007) were reanalyzed. The data were obtained from three subjects in six sessions each, using GE (three sessions) and SE (three sessions) imaging. The target ROI of one subject was unu- 
sually and densely covered by large blood vessels. We therefore excluded the data from this subject and used the two other datasets. A single slice was imaged; it was selected such that it was parallel to and maximally overlapping with a flat gray matter region of the calcarine sulcus. The in-plane resolution was $0.5 \times 0.5 \mathrm{~mm}^{2}$ and the slice thickness was $3 \mathrm{~mm}$. Each run included a baseline epoch, in which a blank gray image was presented, and alternating epochs of left or right eye stimulation. Detailed descriptions of the methods used for data acquisition can be found in Yacoub et al. (2007).

\section{fMRI Data processing}

\section{Data reconstruction}

The measured k-space data were preprocessed using dynamic off-resonance in $\mathrm{k}$-space (DORK) to remove respiration-induced fluctuations in resonance frequency (Pfeuffer et al., 2002). Subsequently, a Fourier transformation was applied in order to transform the data to the image space. Three datasets (subject 2, SE) were acquired using partial Fourier and were reconstructed using a homodyne reconstruction algorithm (Noll et al., 1991).

\section{Motion correction}

Residual head motion was corrected using AFNI's 3dvolreg (Cox and Jesmanowicz, 1999). This algorithm requires multiple slices; therefore, identical copies of the slice were concatenated from above and below. The additional slices were later discarded from the output of the algorithm. The reference volume in each run was set to the volume with the highest average correlation to all other volumes. Each run was motion-corrected using the two-passes option and Fourier interpolation. All volumes for which any voxel was displaced more than $1 \mathrm{~mm}$ relative to the reference volume were marked as motion outliers and were later excluded from the general linear model (GLM) analysis. All resulting transformation matrices were saved.

Between-run motion correction was carried out by first averaging all within-run corrected volumes from each run. Next, the series created from concatenating these single-run averages was corrected in the same manner as described above. Again, all transformation matrices were saved.

Finally, the within-run corrected data was transformed by applying the saved between-run transformations. The resulting combination of two interpolations (within- and between-runs) was created for intermediate use only. For our quantitative analysis of the PSF, only one interpolation was applied. This one interpolation accounted for all alignments and registrations of the data (see below).

\section{Outlier volume detection}

For every volume, the measured fMRI signal in each voxel was compared to the entire time-course of that voxel by computing the z-score of the measured fMRI signal relative to the voxel's time series across all other volumes. Volumes that were already marked as motion outliers were not included in this calculation. The volume under consideration was marked as an outlier volume if the 
average of z-scores across all voxels was larger than 2. Outlier volumes were later excluded from the GLM analysis.

Between-days and between-modality registration

ROIs for each session were imported from our previous analysis of the data (Yacoub et al., 2007). For each modality (GE or SE), between-days registration was performed using mean intensity images averaged over all runs of a given day. First, the single-day ROIs were aligned according to their centers of mass. Next, each day's mean intensity image was cropped such that all images contained the same amount of space around their ROIs, the ROIs were in the same position, and all cropped images were of equal size. Weight masks were calculated for each day by assigning a weight of one to all voxels inside the ROI and zero to all voxels further away from the ROI than $25 \mathrm{~mm}$. Voxels outside of the ROI but closer than $25 \mathrm{~mm}$ were assigned an intermediate weight that varied smoothly between one and zero according to the function $0.5\left(\cos \left(\pi \frac{d_{R O I}}{25 \mathrm{~mm}}\right)+1\right)$, where $d_{R O I}$ is the shortest distance to the ROI. Out of the three days, the one whose mean intensity image had the highest average correlation to those of all other days (weighted by the mask) was selected as the reference day. Each day was registered to the reference day using FSL's flirt 2D registration without large-scale search (Jenkinson et al., 2002; Jenkinson and Smith, 2001), using the weight masks and normalized correlation as the cost function. All transformations were saved.

Initial between-modality (GE and SE) registration was carried out using the registered GE and SE images averaged over all days. A procedure similar to that used for within-modality registration was employed, except that the correlation ratio served as the cost function. For the data of subject 1, AFNI's $3 \mathrm{dvolreg}$ (using the same options as in motion correction) produced a better registration than FSL's flirt based on visual inspection, and was therefore used

All registration results were visually inspected. Residual misalignments found in one day of subject 1 and one day of subject 2 were manually corrected.

\section{Data resampling}

In order to avoid smoothing of the data due to multiple interpolations, all transformation matrices (within-run motion correction, between-run motion correction, between-days within-modality registration and between-modalities registration) were combined. All unprocessed data was transformed using one single Fourier interpolation per volume using AFNI's 3drotate (Cox and Jesmanowicz, 1999).

\section{GLM analysis}

For each run, a GLM was fit to each single voxel time-course. The model consisted of a constant predictor and the two stimulation paradigms (left and right eye stimulation) convolved with a standard hemodynamic response function. Volumes that were previously determined to be outliers due to extensive head motion or imaging artifacts were excluded from the fit. Relative responses were calculated by dividing the estimated stimulus response magnitudes by the estimated constant baseline. Differential responses were calculated as the dif- 
ference between left and right eye responses. Unspecific responses were calculated as the average between left and right eye responses. In addition, standard errors of all estimates were calculated. For visualization purposes only, estimated response maps as well as modeled response maps were interpolated to $0.25 \times 0.25 \mathrm{~mm}^{2}$ resolution by zero-padding in spatial frequency space and were band-pass filtered for eliminating cycles shorter than $1.25 \mathrm{~mm}$ and longer than $12 \mathrm{~mm}$.

\section{Multi-run and multi-day averaging}

Standard errors were comparable between runs and days. Accordingly, singleday responses were calculated by averaging the GLM estimates of the singlerun responses. Likewise, responses for each subject and imaging modality (GE or SE) were estimated by averaging single-day responses.

Standard errors for these averaged responses were estimated as standard errors of the mean from the distribution of single-day responses. Averaged response maps from all three days were used for further processing for subject 1 . For subject 2, between-days correlation of a pair of SE sessions was significantly lower than those obtained from all other pairs in our data. We therefore averaged only the two most reproducible SE sessions (highest correlation of differential responses) and, separately, the two most reproducible GE sessions in order to achieve equal processing between SE and GE.

Optimization of between-modality registration using differential maps

Between-modality registration was further optimized. The GE and SE differential maps were shifted relative to each other vertically and horizontally by multiples of a quarter voxel up to three voxels in each direction and the set of shifts that resulted in the highest correlation between differential GE and SE maps was saved. To avoid multiple interpolations, this shift was combined with all previously found transformations (i.e. motion correction and registration) into a single transformation and interpolation. All unprocessed data were transformed over again as described above, followed by GLM analysis, multirun and multi-day averaging.

\section{Quantities used for MCMC fitting}

Image artifacts, noise and blood vessels may result in some voxels with extreme differential responses that would have a disproportionate effect on fitting the model. For this reason, all voxels with a differential response showing absolute deviation from the median exceeding 3.7 times the median absolute deviation (corresponding to 2.5 standard deviations of a normal distribution; Leys et al., 2013) were excluded (percentage of excluded voxels in subject 1: 5.0\% GE and 4.1\% SE, in subject 2: $2.8 \%$ GE and 2.3\% SE). Note that although this procedure may have removed some voxels with large vessel contributions, it was not meant to systematically remove all voxels with such contributions (see discussion). We then calculated the median unspecific response and the root mean square (RMS) of the differential response standard errors from the remaining voxels. We set the maximum response amplitudes $\beta_{G E}$ and $\beta_{S E}$ to twice the median of the left/right averaged GE and SE responses, respectively, as defined by the model. 


\section{MCMC fitting}

The posterior probability of GE and SE PSF widths given the data and priors over parameters was estimated using MCMC sampling (Fig. 1). MCMC sampling was implemented using a Hamiltonian Monte Carlo algorithm (Duane et al., 1987; see Neal, 2011 for a more recent review).

The algorithm requires input in the form of a function that computes the negative log posterior probability (the potential energy of the model; see Appendix B in the Suppl. Material) and its gradient (see Appendix C in the Suppl. Material). The log posterior probability depends on model parameter values, their prior probabilities, the data and the uncertainty of the data. The data in this sense were the maps of measured differential GE and SE responses within the ROI that were not excluded as outliers. The uncertainty of the data was characterized by the RMS of differential response standard errors, calculated separately for GE and SE. The exact form of the log posterior probability and derivations of the formulae for efficient computation of its gradient are described in the Appendix.

Two parameters determine the dynamics of parameter space exploration. The first parameter, the number of leapfrog steps per iteration, was set to a value of 20. The second parameter, the step size, was initially set to 0.005 and was adjusted adaptively so that the acceptance probability stayed close to the theoretical optimum of 0.651 (Neal, 2011). In addition, the step size was varied randomly within a range of $\pm 20 \%$ to avoid periodicity in the trajectories (Neal, 2011).

Initial values used for all model parameters can be found in Table 1.

The MCMC algorithm was run for 512,000 iterations, of which every $256^{\text {th }}$ sample was retained. The set of all retained samples is an approximation to the joint posterior probability distribution of all parameters given the data and the model, while taking prior distributions into account.

See the attached video, that demonstrates the initial stages of the fitting.

Analysis of MCMC sampling results

The samples of PSF widths were binned into $0.04 \mathrm{~mm}$ wide intervals. We then identified the bin that contained the highest number of samples, which is the maximum a posteriori probability estimate obtained from the marginal distribution for the PSF width. Highest marginal posterior density credible intervals at the $95 \%$ level were computed by selecting the narrowest intervals containing $95 \%$ of the PSF width samples.

MCMC sampling diagnostics

The quality of the MCMC sampling process was assessed by visual inspection of parameter sample traces, autocorrelation estimates of the samples traces and the Geweke diagnostic, which is a z-test for difference between sample means in the first $10 \%$ and last $50 \%$ of samples (Geweke, 1991).

Estimation of $T_{2} / T_{2}^{*}$ blurring or sharpening effect

Imaging modulation transfer functions (MTF) were estimated from the last volume of each run in which the phase-encode gradients were switched off 
(Kemper et al., 2015). This resulted in read-out lines that were expected to vary in amplitude only, according to the phase-encode direction imaging MTF (reflecting $T_{2} / T_{2}^{*}$ decay as experienced by all normally acquired volumes). First, the peak position along the read-out direction was found from the average of the absolute magnitudes computed over all read-out lines. Next, the imaging MTF was estimated by combining the absolute magnitudes of all read-out lines at the peak position into a vector.

We estimated the blurring or sharpening due to $T_{2} / T_{2}^{*}$ decay as a separate effect from the effect of finite and discrete MR sampling (for more details see Chaimow and Shmuel, 2016). To this end, the complex imaging PSF was computed by applying a discrete Fourier transform to the estimated imaging MTF.

Convolution of the original pattern with the real component of the complex imaging PSF is an approximation to the full MRI acquisition (Chaimow and Shmuel, 2016), including the last stage of taking the absolute of the complex values obtained at the end of the reconstruction.

We computed the inverse discrete Fourier transform of the real component of the complex imaging PSF, resulting in its MTF. Two Gaussian functions with zero means were separately fitted to the MTF of the real component of the complex PSF and to its inverse. Then, we compared the goodness of fit $\left(\mathrm{R}^{2}\right)$ obtained by the two fitted Gaussians. If the better fit was obtained by fitting a Gaussian to the MTF of the real component of the complex PSF, the effect of $T_{2} / T_{2}^{*}$ could be described as Gaussian blurring. If the better fit was obtained by fitting a Gaussian to the inverse of the MTF of the real component, the effect of $T_{2} / T_{2}^{*}$ could be described as a sharpening that could reverse a specific Gaussian blurring.

We therefore computed the FWHM of the Gaussian with the better goodness of fit (obtained by fitting to either the MTF of the real component or to its inverse).

Note that in the case of sharpening, the computed FWHM characterizes the Gaussian 'used' for blurring which would be reversed by the sharpening effect of the $T_{2} / T_{2}^{*}$ decay. FWHM estimates for each modality were first averaged over all runs of each session (day) and then over all sessions of each subject.

\section{Inclusion of $\mathrm{T} 2 / \mathrm{T} 2 *$ blurring in the model}

A version of our model that included the effect of $T_{2} / T_{2}^{*}$ decay was fitted to our data. Depending on whether the $T_{2} / T_{2}^{*}$ decay effect resulted in blurring or sharpening, the BOLD MTF (Appendix A, BOLD response) was changed to: $M T F_{k, l}(f w h m, \beta)=\beta \cdot e^{-2 \pi^{2} \sigma_{B O L D}^{2} \cdot r(k, l)} \cdot e^{-2 \pi^{\wedge} 2 \cdot \sigma_{I M G}^{2} \cdot l^{2}} \quad$ (for blurring) or $M T F_{k, l}(f w h m, \beta)=\beta \cdot 1 / e^{-2 \pi^{2} \sigma_{B O L D}^{2} \cdot r(k, l)} \cdot e^{-2 \pi^{\wedge} 2 \cdot \sigma_{I M G}^{2} \cdot l^{2}}$ (for sharpening), where $\sigma_{I M G}=f w h m_{I M G} / 2 \sqrt{2 \log 2}$ and $f w h m_{I M G}$ is the estimated FWHM of the Gaussian blurring kernel that models the effect of $T_{2} / T_{2}^{*}$ decay. We assumed the second dimension (associated with index $l$ ) to be the phase-encode dimension. 


\section{Results}

Our goal was to fit a probabilistic generative model to maps of ODCs obtained with GE- and SE-based BOLD fMRI (Fig. 1). We aimed to infer posterior probability distributions of model parameters, specifically the width of the GE and SE BOLD fMRI PSF.

\section{Parameter priors obtained from real human ODC}

The ODC imaging model (Chaimow et al., 2011) consisted of simulating realistic ODCs by the filtering of spatial white noise (Rojer and Schwartz, 1990) followed by a spatial BOLD response and MRI k-space sampling.

Before we fitted our model to fMRI data, we determined priors for the ODC parameters by incorporating statistical information obtained from real human ODC patterns (Fig. 2). To this end, we analyzed CO maps of ODCs from human V1 taken from Adams et al. (2007).

It should be noted that CO labeling intensities are expected to provide a fairly accurate estimate of the preferred eye. However, there are multiple, potentially non-linear transformations between neuronal activity, staining intensity and the final processed image. These make it unlikely that the CO intensities quantitatively reflect the relative ocular dominance. Therefore, we only used binarized versions of these maps, thresholded to represent the absolute preference to either left or right eye stimulation). We eventually determined priors on ocular dominance from neurophysiological recordings (Berens et al., 2008; Hubel and Wiesel, 1968).

We first restricted the maps of the entire V1 to small regions (Fig. 3A) whose size and location were similar to those of our fMRI data (with origins in flat regions of the calcarine sulcus). Then, for each map, we fitted the parameters of the ODC part of the model such that the spatial power spectra of the simulated binarized ODC maps were similar to those of the CO maps of ODCs (Fig. 3B, measured ODC; Fig. 3C, simulated ODC). Simulated maps generated using these parameters looked qualitatively similar to the true CO maps (Fig. 3C). The set of all imported maps is shown in Figure 3D and the estimated parameters from all maps are shown color-coded in Figure 3E.

For each parameter we defined Gaussian priors that fit the distribution of all remaining parameter estimates (black curves, Fig. 3E). In particular, the prior for the main pattern frequency $\rho$ had a mean of 0.57 cycles $/ \mathrm{mm}$ with a standard deviation of 0.1 cycles $/ \mathrm{mm}$, which corresponds to an average column width of $0.87 \mathrm{~mm}$.

\section{Smoothness of ODC maps}

In order to construct a prior for the smoothness parameter $\omega$ we analyzed distributions of ocular dominance indices (ODIs) as reported in the neurophysiological literature. ODIs quantify the relative contributions of each eye to measured responses, and their distribution is tightly linked to the smoothness parameter $\omega$. Small values of $\omega$ result in sharp transitions between columns associated with ODIs close to +1 or -1 . Large values of $\omega$ result in smooth transi- 
tions, with few locations reaching absolute monocular responses and most ODIs being close to 0 .

We analyzed ODI distributions taken from Hubel and Wiesel (1968) and Berens et al. (2008) by fitting ODI distributions computed from our model as a function of smoothness $\omega$. We found a value of $\omega=1.5$ to best explain ODI distributions corresponding to the data in Berens et al. (2008), whereas the data in Hubel and Wiesel (1968) were best fitted with $\omega=0.36$. Both datasets came from macaque monkeys. Berens et al. (Berens et al., 2008) used multiunit activity, a measure whose ODIs are expected to be blurred relative to single neuron responses and are therefore expected to match a higher $\omega$. Data from Hubel and Wiesel (Hubel and Wiesel, 1968) presented single-unit responses but were less quantitative. We therefore chose a uniform prior distribution for $\omega$, limited by 0.3 from below and 2 from above, effectively reflecting the range of uncertainty associated with $\omega$.

\section{GE and SE BOLD maps of ODC}

Having constructed a generative model with realistic priors, the next step was to process the fMRI data and to extract all quantities needed to fit the model.

We reanalyzed fMRI data from two subjects (Yacoub et al., 2007) using a general linear model (GLM) to estimate responses to left and right eye stimulation (Fig. 4). The single-eye response maps were dominated by global unspecific responses and superimposed band-shaped modulations (Fig. 4A).

We separated these two components by first calculating the voxel-wise difference between left and right eye responses, yielding the differential ODC maps (Fig. 4C). Here, the band-shaped organization is clearly visible. The range of differential contrasts as defined by their standard deviation was $1.8 \%$ (GE) and 1.5\% (SE) for subject 1, and 1.0\% (GE) and 1.0\% (SE) for subject 2.

In addition, we calculated voxel-wise averages of left and right eye responses (Fig. 4B). According to our model, which assumes antagonistic patterns of neuronal responses, this average response is expected to be independent of the local ocular preference. Furthermore, it is expected to be equal to a spatially homogeneous response with half the amplitude of the highest possible ocular dominance (with no response to the non-preferred eye).

We calculated the median of this left/right average response over all voxels. It was 3.0\% (GE) and 1.9\% (SE) for subject 1 , and $3.7 \%$ (GE) and $2.0 \%$ (SE) for subject 2 . In accordance with the model, we then set the amplitudes of the model PSFs to twice these values.

Finally, we estimated the measurement noise level of the differential maps as the root mean square (RMS) of all standard errors estimated by the GLM (Fig. 4D). It was $0.9 \%$ (GE) and $0.9 \%$ (SE) for subject 1 , and $0.6 \%$ (GE) and $0.8 \%$ (SE) for subject 2 . 


\section{GE}

A

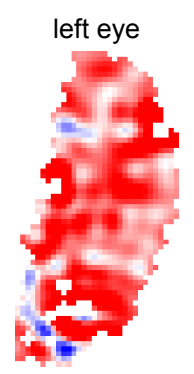

B

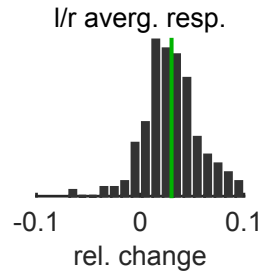

C
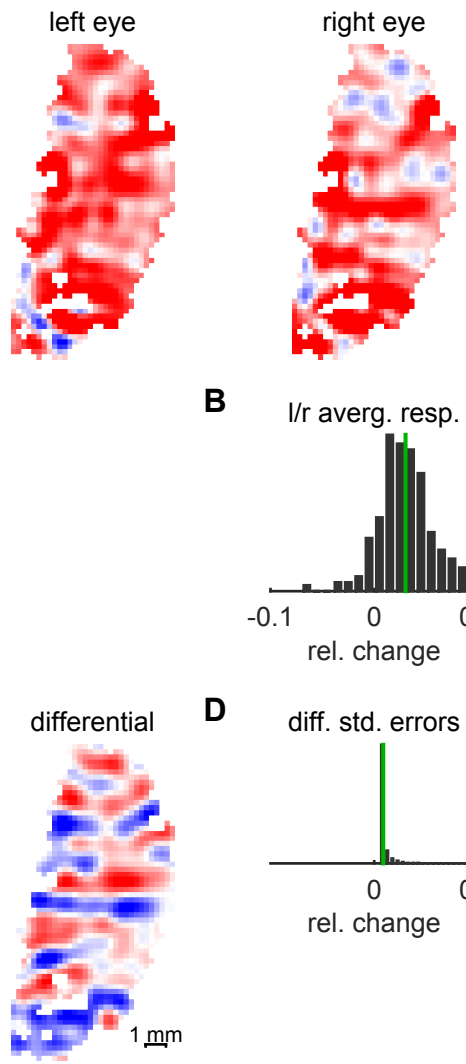

SE

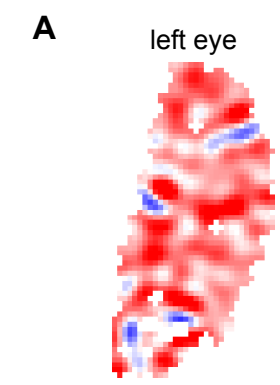

B

C

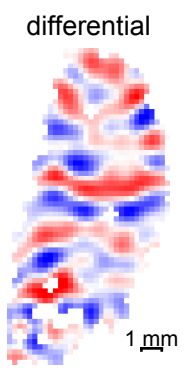

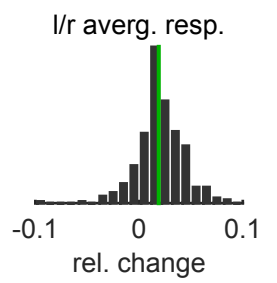

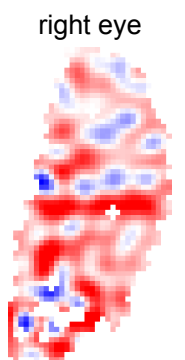

D

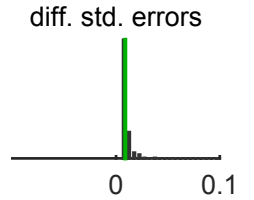

rel. change

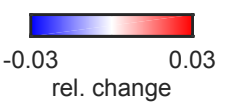

Fig. 4 fMRI ODC data. Results from the GLM analysis of fMRI data from subject 1 for GE (left) and SE (right). A Responses to left and right eye stimulation relative to baseline. B The response maps to the left and right eyes from A were averaged. B shows the distribution of the average response. Its median (in green) was used to set the overall amplitude of the BOLD response model. C The difference between left and right eye responses yields the differential ODC map. D The distribution of standard errors of all differential responses. From this distribution we estimated the noise level used by the model. The color look-up-table applies to all response maps.

\section{Estimation of GE and SE point-spread widths}

We went on to estimate the probability distributions of GE and SE PSF widths given our data. Theoretically, this requires integrating the posterior probability distribution of model parameters over all other parameters (including the high-dimensional spatial noise parameter). However, exact integration over this high dimensional space is not feasible. We therefore used MCMC to sample from the posterior probability distribution. Every sample contains all the parameters necessary to simulate one anatomical ODC map and the resulting GE and SE fMRI differential maps. The algorithm draws parameter samples with a probability proportional to how likely these parameters are to have generated the measured data given all the priors. 
S1
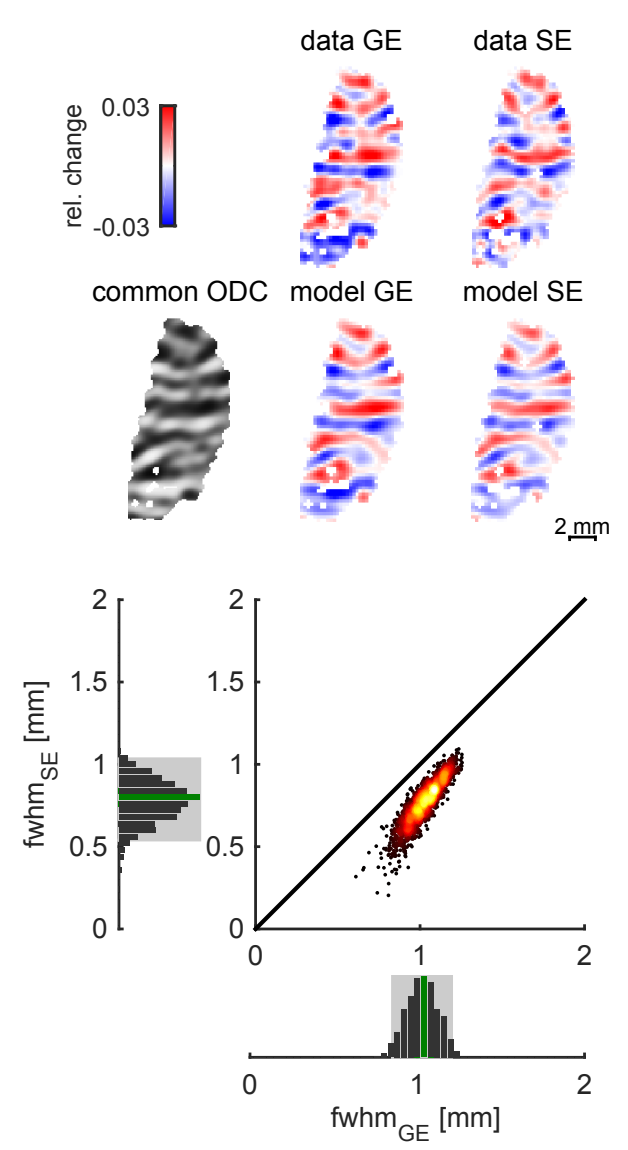

S2
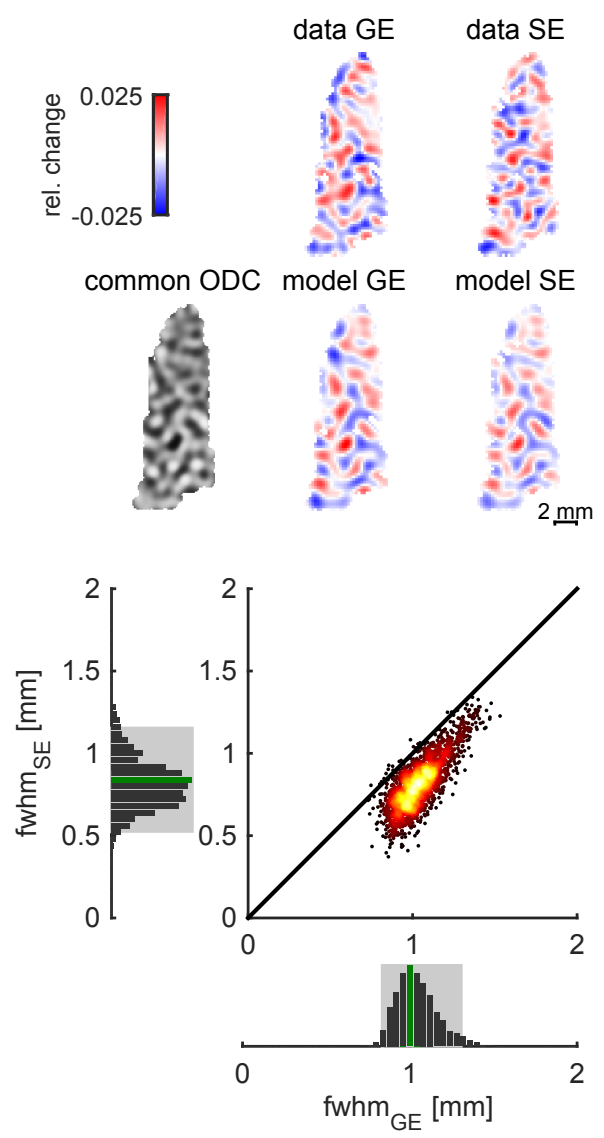

Fig. 5 Results of point-spread width estimation. The probability distribution of PSF given the data was estimated using Markov Chain Monte Carlo Sampling. The model simulated GE and SE maps each with their own BOLD parameters and with a common underlying ODC map. Results are shown for both subjects. The first row shows the measured differential ocular dominance map from the GE (left) and SE (right) experiments. The second row shows the modeled underlying ODC maps (left) and the modeled differential fMRI maps from the maximum a posteriori (MAP) sample. The bottom part of the figure shows the joint and marginal distributions of GE and SE point-spread full-widths at half-maximum (FWHM). The gray rectangles show the 95\% credible intervals (highest posterior density interval). The scatter plots show that the vast majority of individual GE PSF samples were wider than their SE PSF counterparts. The MAP estimates (green bars) obtained from the marginal distributions of the FWHMs of the PSFs were 1.04 and $1.0 \mathrm{~mm}$ (GE), and 0.8 and $0.84 \mathrm{~mm}$ (SE) for subjects 1 and 2 respectively.

Figure 5 (second row, common ODC) shows one of many possible ODC patterns generated by our model. It was generated using the parameter sample with the highest posterior probability. Differential BOLD fMRI maps modeled as arising from this shared ODC pattern (Fig. 5 second row, model GE and model SE) resemble the data closely (Fig. 5 first row, data GE and data SE). The distribution of PSF widths from all samples (Fig. 5 bottom) is an estimate of the true probability distribution of PSF widths for that data (see the attached video that demonstrates the initial iterations of the fitting procedure). 


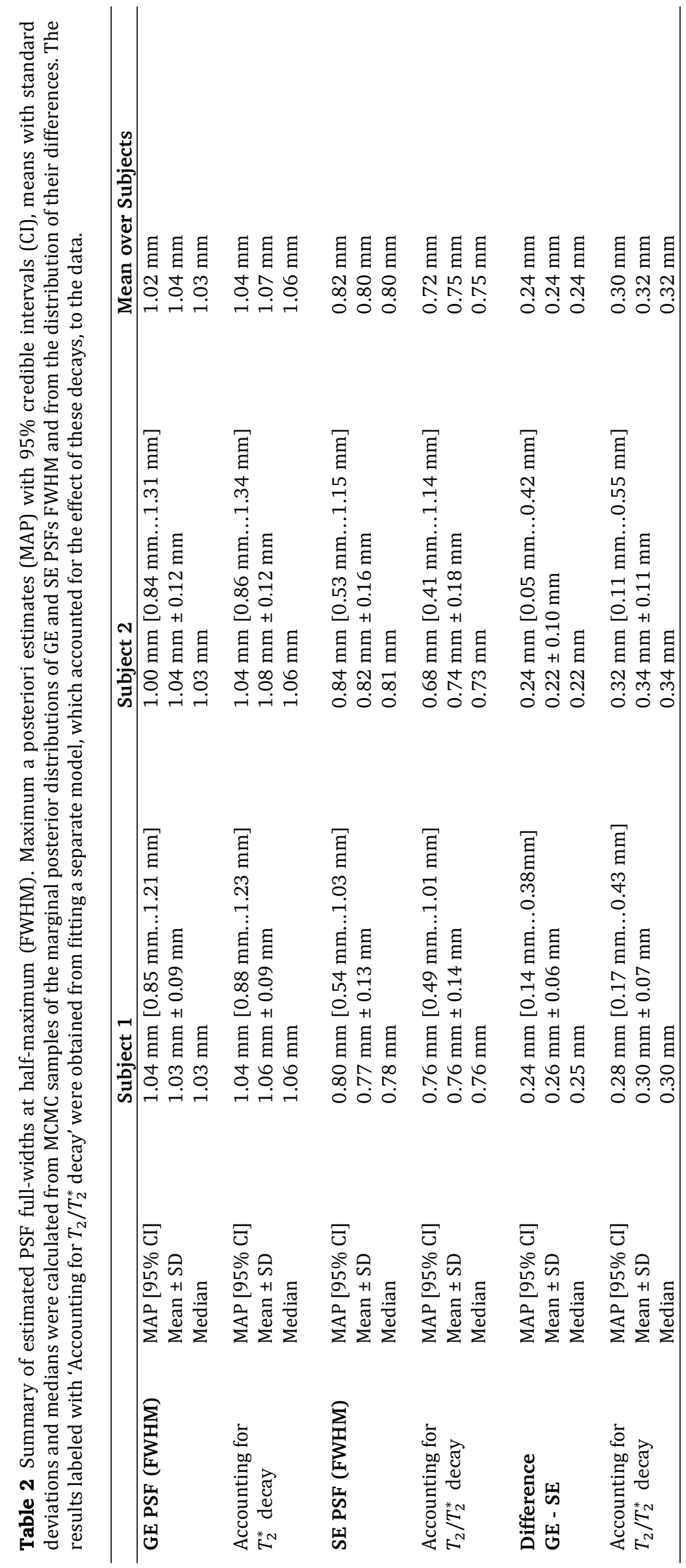


Figure 5 (bottom) and Table 2 present the results of PSF widths. For subject 1 , the most probable (maximum a posteriori estimate obtained from the marginal distribution of FWHMs) GE PSF width was $1.04 \mathrm{~mm}$ (FWHM), with $95 \%$ of the values falling between $0.85 \mathrm{~mm}$ and $1.21 \mathrm{~mm}$. The most probable SE PSF width was $0.80 \mathrm{~mm}$, with $95 \%$ of the values falling between $0.54 \mathrm{~mm}$ and $1.03 \mathrm{~mm}$. For subject 2, the most probable GE PSF width was $1.00 \mathrm{~mm}$, with $95 \%$ of the values falling between $0.84 \mathrm{~mm}$ and $1.31 \mathrm{~mm}$. The most probable SE PSF width was $0.84 \mathrm{~mm}$, with $95 \%$ of the values falling between $0.53 \mathrm{~mm}$ and $1.15 \mathrm{~mm}$.

Furthermore, the samples of GE and SE PSF widths were correlated. This means that ODC model parameters that resulted in a relatively higher GE PSF width also resulted in a relatively higher SE PSF width. Across all modeled underlying anatomical ODC patterns, the GE PSF was almost always wider than the SE PSF. We calculated the resulting posterior distribution of differences between GE and SE PSF widths. The bottom part of Table 2 summarizes the estimated differences for the two subjects. For subject 1 , the most probable difference was $0.24 \mathrm{~mm}$, with $95 \%$ of the values falling between $0.14 \mathrm{~mm}$ and $0.38 \mathrm{~mm}$. The most probable difference obtained for subject 2 was $0.24 \mathrm{~mm}$, with $95 \%$ of the values falling between $0.05 \mathrm{~mm}$ and $0.42 \mathrm{~mm}$.

\section{Evaluation of model fit}

The validity of our results depends on how well the MCMC samples approximate the target distribution. The MCMC sampling distribution approaches the target distribution when the number of iterations goes to infinity (e.g. see Neal, 1993). For sufficiently large number of iterations, MCMC effectively samples from the target distribution.

While there cannot be proof that the target distribution has been reached, there are a number of indications that are considered reliable. The first is that the traces of samples of all parameters have settled into a stationary distribution, with no slow drifts over iterations. This can be seen in the single parameter trace plots (Fig. 6, first column) and their autocorrelation plots (Fig. 6, second column). In addition, the Geweke diagnostic (Geweke, 1991) shows that for all single parameters the mean of the first $10 \%$ of the samples was not significantly different from the last $50 \%$ of the samples $(|z|<1.96)$. The Geweke diagnostics for the high-dimensional noise follow a standard normal distribution (Fig. 6, bottom, distribution of z-scores), as would be expected by chance under the hypothesis that the means are not different. Figure 6 also shows the dependences between PSF widths and ODC parameters (last two columns). As can be seen, higher levels of smoothness parameter $(\omega)$ values and to a lesser extent lower levels of the main spatial frequency parameter $(\rho)$ values made a narrower PSF more likely. 
bioRxiv preprint doi: https://doi.org/10.1101/097287; this version posted December 29, 2016. The copyright holder for this preprint (which was not certified by peer review) is the author/funder, who has granted bioRxiv a license to display the preprint in perpetuity. It is made available under aCC-BY-NC-ND 4.0 International license.

\section{A Parameters}
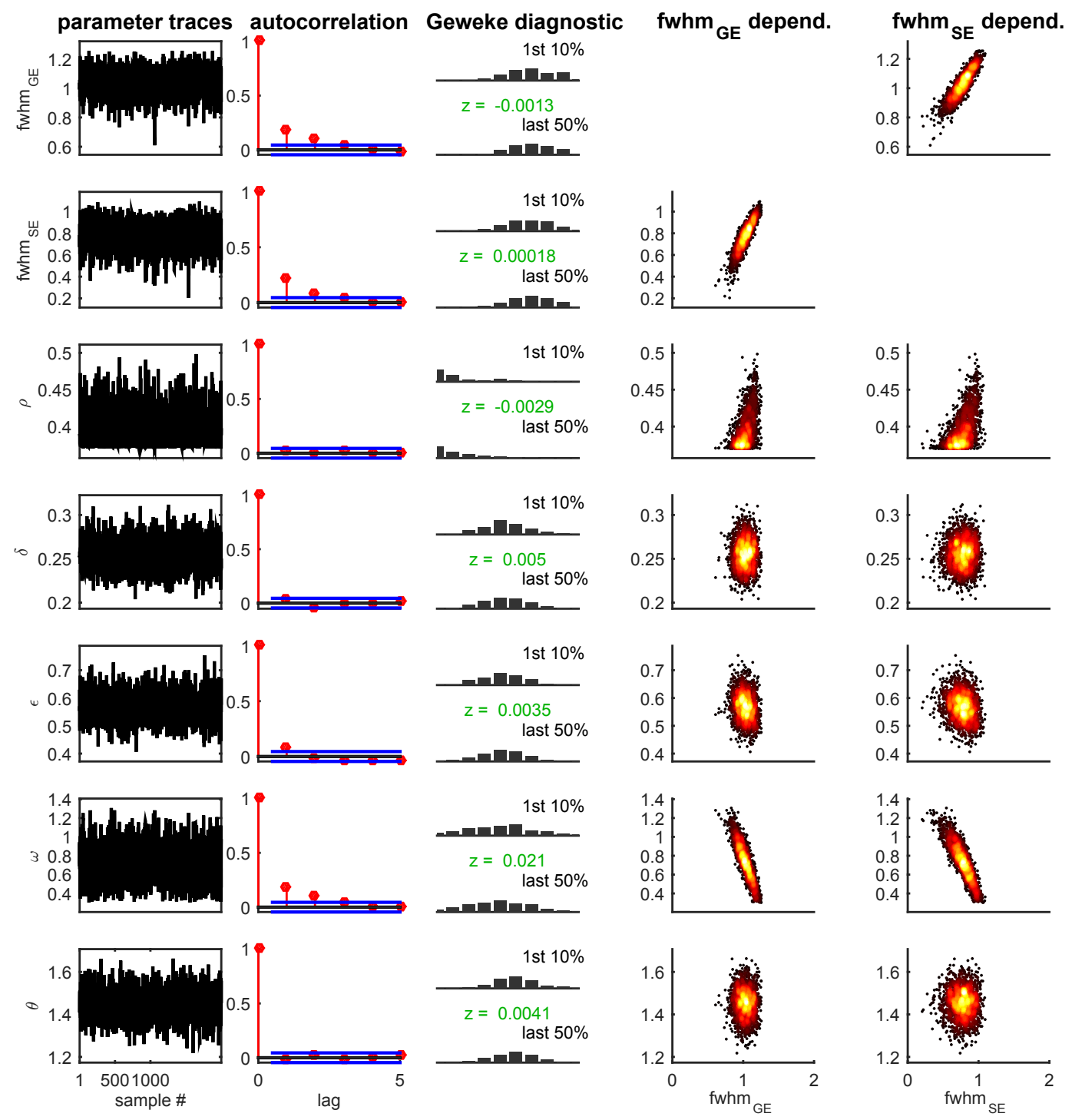

\section{B Noise values}
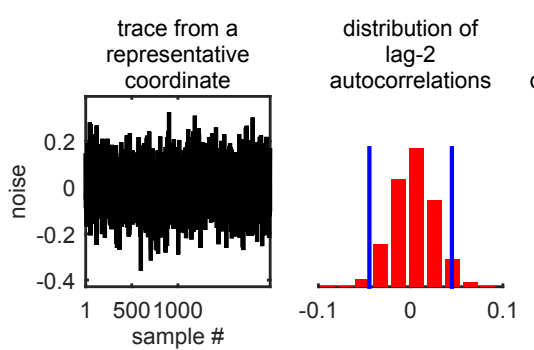

distribution of z-scores

Fig. 6 Convergence diagnostics of Markov Chain Monte Carlo sampling. Markov Chain Monte Carlo needs to run for a sufficient number of iterations in order to yield samples from the modeled probability distribution. Indications for convergence are: (1) stationarity of the parameter sampling distributions, and (2) sample autocorrelations decrease rapidly with increasing lag, relative to the total number of samples. This figure examines convergence for subject 1 . The upper part of the figure (A) shows diagnostics for the standard model parameters. The bottom part (B) shows diagnostics for the white noise values that act as parameters to determine the ODC pattern. The first column (A and B) shows traces of the sampled parameters. For the noise values (B), one exemplary trace is shown from the center of the map. The second column (A and B) shows sample autocorrelations as a function of lag. The horizontal blue lines (A) indicate the 95\%-confidence bounds around 0 for a white noise process. Consecutive samples $(\mathrm{lag}=1)$ show low autocorrelation. However, samples of lag 2 (and higher) show autocorrelation esti- 
mates that are comparable to those obtained from uncorrelated white noise. In $\mathrm{B}$, the autocorrelation from the noise samples are summarized by the histogram of lag- 2 autocorrelations from all coordinates together. Here, 95\%-confidence bounds for a white noise process are indicated by vertical blue lines. The third column (A and B) presents the Geweke convergence (stationarity) diagnostic, which is a z-test (zscores shown in green) for testing whether the means of the first $10 \%$ and last $50 \%$ of samples are different. In A, 2 histograms per each parameter show how similar their respective distributions are. In B, the z-scores from the noise samples are shown as a histogram together with a blue plot of the standard normal probability density representing the null-hypothesis of $\mathrm{z}=0$. The last two columns (A) show the sample covariation between each parameter (vertical axis) and the GE and SE point-spread function FWHM (horizontal axis).

\section{Discussion}

Possible confounds: our estimates are upper bounds of BOLD fMRI spatial specificity

The PSF widths that we estimated (1.02 $\mathrm{mm}$ for GE BOLD, $0.82 \mathrm{~mm}$ for SE BOLD) reflect the realistically achievable spatial specificity of BOLD signals at ultra-high field strength (7T). However, they are only upper bounds for the true BOLD PSF widths. Subjects' head motion, data interpolation and intraacquisition $T_{2} / T_{2}^{*}$ decay can all introduce additional blurring (but see section below on the effect of $T_{2} / T_{2}^{*}$ decay), causing the estimated PSF to be wider than the true PSF.

In order to minimize head motion, data was acquired from trained subjects using a bite bar. Before each scan, the position of the region of interest (ROI) was checked and the slices repositioned if necessary. We corrected the data for residual head motion and discarded any problematic volumes. We aligned data from multiple days and checked the alignment carefully. In order to further optimize between-modality registrations, we also took the differential fMRI response patterns into account, making use of the fact that they emerged from the same underlying neuronal ODC pattern.

Motion correction and between-day registration required spatial interpolation of the data. We minimized any blurring effects by applying all spatial transformations combined using one single Fourier interpolation (Cox and Jesmanowicz, 1999).

All high spatial resolution BOLD fMRI experiments will be influenced by these effects to a similar degree as ours, making our reported PSF widths good estimates for the practically relevant compound effect.

\section{Possible confounds: the contribution of the imaging PSF to the total BOLD fMRI PSF}

In addition to the effects of the hemodynamic and metabolic responses on the spatial specificity of fMRI, the MRI acquisition process influences the effective resolution of the acquired images. Specifically, the sampling of k-space by means of temporal gradient encoding defines the spatial resolution. However, the effective spatial resolution along the phase encoding direction in EPI acquisitions can be subject to blurring or sharpening, because of $T_{2} / T_{2}^{*}$ decay while the k-space is being sampled. This can potentially contribute to the overall measured spread of the BOLD fMRI signal. 
In order to minimize this effect, our data were acquired using a reduced field-of-view (in SE) and multiple segments. These measures limited the total read-out duration per segment ( $25.6 \mathrm{~ms}$ for GE and $24 \mathrm{~ms}$ for SE) to approximately the $T_{2}^{*}$ of the tissue (Uludağ et al., 2009) and are expected to result in only minor blurring or sharpening (Haacke et al., 1999)).

We estimated the blurring or sharpening and their contributions to the total BOLD PSF. In general, MRI data acquisition using EPI has two distinct effects on the effective spatial resolution. The first is the effect of the finite and discrete MR sampling with no decay. However, in the current study MR sampling was part of the model and therefore has already been accounted for. The second effect is the already mentioned $T_{2} / T_{2}^{*}$ decay blurring or sharpening. This effect is limited to the phase encode direction (vertical direction in all presented maps). In order to characterize it, we estimated the imaging modulation transfer functions (MTF) along the phase encoding direction from a reference volume obtained in each run, in which the phase-encode gradients were switched off (Kemper et al., 2015).

Using a model of Gaussian convolution and MRI sampling (Chaimow and Shmuel, 2016, in preparation) we obtained Gaussian functions that can model the separate effect of $T_{2} / T_{2}^{*}$ decay. We then used MCMC to fit a separate version of our model to our data, where we applied the decay effect to the simulated ODC patterns by modulating the values acquired in the simulated kspace. The results presented in Table 2 show that the effect of the signal decay on the total BOLD PSF was small. For GE, while accounting for the signal decay, we obtained PSFs wider than the effective PSF obtained directly from the BOLD fMRI response. This indicates that signal decay in the GE fMRI used for obtaining our data has a sharpening effect. In contrast, the signal decay in the SE fMRI used for obtaining our data has a blurring effect. These results demonstrate that the physiological BOLD response measured with GE fMRI (that theoretically does not include signal decay) is less spatially specific than the same physiological BOLD response measured with SE fMRI (with no signal decay). This difference in spatial specificity of the GE and SE BOLD responses (with no signal decay) is even slightly larger than the corresponding effective difference obtained from the overall measured fMRI responses with signal decay.

\section{What do our estimated point-spread function widths describe?}

The BOLD PSF describes the spatial specificity of the BOLD fMRI signal by characterizing the spatial response that would be elicited by a small point stimulus. Specifically, our BOLD PSF width measures the spread of the BOLD fMRI response (I) elicited by a small spot of neuronal activity, (II) along the cortical manifold, (III) using a differential response analysis, (IV) assuming that in a differential analysis paradigm the average spread can be described by a Gaussian function, and (V) considering a relatively long time scale.

(I) BOLD PSF relative to the local neuronal activity

To the best of our knowledge, our PSF estimates are the first to quantify the BOLD spread in human subjects relative to local neuronal activity. We previ- 
ously estimated the FWHM of the 7 T GE BOLD PSF to be smaller than $2 \mathrm{~mm}$ by measuring the spread of the BOLD fMRI response around the V1 representation of edges of visual stimuli (Shmuel et al., 2007). We expect that our previous estimates as well as others' (Engel et al., 1997; Parkes et al., 2005) included contributions from non-zero extent of receptive fields and the scatter of receptive field position of neurons in $\mathrm{V} 1$.

Hubel and Wiesel (1974) reported that in the macaque "... a $2 \mathrm{~mm} \times 2$ mm block of cortex contains the machinery needed to analyze a region of visual field roughly equal to the local field size plus scatter"'".

These observations suggest that visual stimuli will result in neuronal activity that is blurred on the surface of human V1. All PSF widths that have been estimated using spatial representations of visual stimuli included this neuronal spread by nature of their experimental design. In the current study, we instead used a spatial structure of neuronal responses that is inherent to the cortexODC patterns. This allowed us to estimate a PSF that does not contain contributions from the spatial spread of responses to visual stimuli.

There are a number of measures of neuronal activity that a BOLD PSF could potentially relate to, notably single-unit activity (SUA), multi-unit activity (MUA) and local field potentials (LFP). Under specific circumstances, these measures can show very different activity. Under most conditions, however, they are highly correlated. This is likely to be true when mapping a cortical columnar organization. The main difference is that the spatial extent (that influences the smoothness of the spatial response pattern) of these signals increases from SUA to MUA to LFP. We estimated a smoothness prior using ODI distributions of SUA and MUA. Consequently, our PSF is based on these signals. The BOLD PSF from LFP would be narrower than our estimate because of the wider cortical spread of LFP compared to MUA activity (Xing et al., 2009).

(II) Spatial BOLD response along the cortical manifold

It has been demonstrated (Polimeni et al., 2010) that the PSF consists of different radial and tangential components relative to the cortical surface. The radial component describes the spread across cortical layers while the tangential component describes the spread parallel to the cortical surface. Here we investigated the tangential PSF, averaged over all layers. This is the component that is most relevant for imaging the representation of cortical columns parallel to the cortical surface. Accordingly, the location and orientations of voxels, the ROI, and the voxel size were all optimized to sample gray matter tangentially and to obtain an average from all layers.

It should be noted that there are some differences in cerebrovascular organization with respect to radial and angular direction (Duvernoy et al., 1981). The largest blood vessels are the pial surface veins that extend in various orientations along the tangential plane. Somewhat smaller are corticalpenetrating veins that are organized radially, traversing the different cortical layers. The smallest vessels, the capillaries, form a fine mesh that locally appears to be isotropic. However, their density varies with cortical layers (Weber et al., 2008). For these reasons, we cannot directly apply our PSF to the imaging of cortical layers. In addition, the distinctiveness and finite extent of layers 
appear to make a PSF convolution model ill-suited for fMRI of cortical layers. However, some recent results (De Martino et al., 2015; Fracasso et al., 2016; Muckli et al., 2015; Olman et al., 2012) suggest it is possible to differentially resolve layer-specific signals on the scale of $1 \mathrm{~mm}$ or less.

(III-IV) On modeling the average differential BOLD response as a Gaussian PSF We assumed the average (over space) PSF to be a Gaussian function. However, the shape of the spread in specific cortical locations may be more complex and location-dependent (Kriegeskorte et al., 2010; Polimeni et al., 2010). Also, its width as well as the magnitude of the response may vary due to local variations in vascular geometry. In fact, the relatively wide distribution of average responses in our data (Fig. 4, distribution of $1 / \mathrm{r}$ averg. resp.) supports this latter intuition. Therefore, a convolutional model with a single Gaussian function can only be an approximating simplification. Nevertheless, we believe that such a simplifying approach provides a useful approximation for planning and interpretation of high-resolution fMRI studies and for quantitative modeling.

As part of our pre-processing before fitting the model to the data, we removed $1.5-5.0 \%$ of voxels that had extreme differential values (see methods section for precise criterion for exclusion). Part of these voxels were located in areas that were previously shown to contain blood vessels (Shmuel et al., 2010). However, for our current analysis we did not explicitly and systematically remove voxels that were affected by larger blood vessels. Our reasoning was that a consistent removal of all voxels suspected to be influenced by larger blood vessels would have reduced contiguous areas of ODCs, which would have made the model fitting more difficult.

We expect the influence of geometric variations in local vasculature to be higher for veins and venules than for capillaries because of their respective diameters and densities. Consequently, the GE BOLD signal, which is more sensitive to larger pial surface veins will be more affected by these local variations. As a result, GE BOLD imaging does not only suffer from a slightly wider PSF than SE BOLD, but it is also subject to local distortions when larger blood vessels are present.

However, although draining veins may show responses with a preference to a subset of features encoded in a columnar organization (Shmuel et al., 2010), differential analysis reduces contributions from macroscopic vessels because of their tendency to drain blood from a region larger than that of a small number of columns. Taken together, a Gaussian PSF model by itself is likely not a good model for single-condition imaging when influenced by large blood vessels (e.g. in GE BOLD imaging). In contrast, we expect that a Gaussian PSF is a good model in a differential analysis paradigm, which reduces contributions from macroscopic vessels. The BOLD PSFs we report here reflect the spatial specificity that can be achieved in a differential paradigm. They do not reflect the spatial specificity expected from single-condition imaging that involves contributions from macroscopic vessels, such as single-condition GE fMRI and to a lesser extent, single-condition SE fMRI. 
(V) Spatial specificity as a function of stimulus duration

It has been shown that the early phase of the positive BOLD response (up until $\sim 4 \mathrm{~s}$ after stimulus onset) is spatially more specific than the later phase (Goodyear and Menon, 2001; Shmuel et al., 2007). On the other hand, stimulation paradigms that use very brief stimulation durations suffer from a highly reduced contrast-to-noise ratio, because the response does not develop to its highest potential amplitude.

We found previously that after $4 \mathrm{~s}$ the spatial BOLD response remained stable and that the entire spatiotemporal response could be well approximated by the first spatial principle component (Shmuel et al., 2007). Aquino et al. (2012) modeled the BOLD response as a travelling wave evolving in time and found that deconvolution of neural dynamics using such a model resulted in physiologically more plausible spatiotemporal patterns than when using a model separable in space and time (Aquino et al., 2014). The spatial profile alone, however, was very similar for both models.

Taken together, long stimulation paradigms are an efficient way of highresolution imaging and their spatial PSF can be well described by a single time-independent component. The stimulation periods for our data were $48 \mathrm{~s}$ long, thereby making our PSF most applicable to long stimulation paradigms.

\section{Spatial specificity of the BOLD response}

Constraints on the spatial specificity of BOLD

The positive BOLD signal depends on decreases in deoxyhemoglobin content in the capillaries which then propagate downstream to draining venules and veins. These decreases are caused by elevated cerebral blood flow (CBF) and only smaller fractional increases in the oxygen consumption rate, following increases in neuronal activity. CBF is regulated at a sub-millimeter scale: (Duong et al., 2001). Similarly, Vazquez et al. (2014) reported a spread of cerebral blood volume (CBV) of $103-175 \mu \mathrm{m}$ (FWHM) in mice using optical imaging. Although this measure is not directly comparable to the CBF spread in a different species (human subjects), it demonstrates that hemodynamic signals can show very high spatial specificity. The CBF response is the ultimate lower limit for the spatial specificity of any BOLD-based technique.

The deoxyhemoglobin content changes in the draining venules and veins are ultimately diluted downstream, because the draining veins pool blood not only from active but also from non-active regions. For an activated area of 100 $\mathrm{mm}^{2}$, Turner et al. (2002) estimated the maximal extent of undiluted oxygenation changes along a vein to be $4.2 \mathrm{~mm}$. For these reasons, we can expect the PSF width of any BOLD-based imaging technique to fall in this range; that is, less than $1 \mathrm{~mm}$ (Duong et al., 2001) to approximately $4.2 \mathrm{~mm}$ (Turner, 2002). The values will be determined by how much weighting towards the microvasculature can be achieved and on the actual presence of larger draining veins in the region of interest.

PSF dependence on field strength

At standard magnetic fields, the width of the BOLD PSF has been estimated to be $3.5 \mathrm{~mm}$ for $1.5 \mathrm{~T}$ GE BOLD (Engel et al., 1997), $3.9 \mathrm{~mm}$ for $3 \mathrm{~T}$ GE BOLD 
and $3.4 \mathrm{~mm}$ for $3 \mathrm{~T}$ SE BOLD (Parkes et al., 2005). These estimates of PSF widths were confounded by the above described receptive field and scatter effects. We can make a rough estimate of what the non-confounded PSF widths at lower fields would be. We assume that on average the receptive field effect can be modeled as another convolution with a Gaussian. It follows that the square of the confounded PSF width is equal to the sum of squares of the receptive field effect width and the non-confounded PSF width. For the receptive field effect we get an FWHM of $2.12 \mathrm{~mm}$ when using $2.35 \mathrm{~mm}$ as the $7 \mathrm{~T} \mathrm{GE}$ BOLD confounded PSF width (Shmuel et al., 2007) and $1.02 \mathrm{~mm}$ as the corresponding non-confounded PSF width (results from our current study). This in turn results in non-confounded estimates of $2.8 \mathrm{~mm}$ (1.5 T GE BOLD), $3.3 \mathrm{~mm}$ (3 T GE BOLD) and $2.7 \mathrm{~mm}$ (3 T SE BOLD).

These PSF widths are considerably larger than the estimates from the current study (1.02 $\mathrm{mm}$ for 7T GE BOLD, $0.82 \mathrm{~mm}$ for 7T SE BOLD). The reason for this is that the BOLD signal (both GE and SE BOLD) at lower field strengths is dominated by intravascular signals from draining veins (Jochimsen et al., 2004; Uludağ et al., 2009). At higher field strengths, the contributions from intravascular signals are reduced due to a shortening of the venous blood $T_{2}$. In parallel, the relative contributions of extravascular signals around small vessels increase (Duong et al., 2003; Uludağ et al., 2009; Yacoub et al., 2003; 2001).

All PSF widths from field strengths of up to $3 \mathrm{~T}$ appear to fall close to the wider end of possible PSF widths. In contrast, PSF widths using SE and GE at 7 $\mathrm{T}$ appear close to their theoretical minimum.

$\boldsymbol{T}_{\mathbf{2}}^{*}$ and $\boldsymbol{T}_{\mathbf{2}}$ based imaging methods: GE, SE and GRASE

We found the SE BOLD PSF to be narrower than the GE BOLD PSF. This is expected because the refocusing pulse in SE imaging suppresses the extravascular signal around larger blood vessels while leaving the signal around the microvasculature intact. As a result, compared to GE BOLD fMRI, SE BOLD signals obtained at 7T have relatively larger contributions from the spatially more specific microvasculature, whereas at lower field strength the signal of either SE or GE BOLD fMRI is dominated by intravascular contributions of large blood vessels.

However, the suppression of extravascular signal around larger blood vessels by SE at high fields is not perfect. Only the k-space data that is sampled at the exact echo time will result in absolute suppression (pure $T_{2}$ weighting as compared to $T_{2}^{*}$ weighting). The extent to which sampled k-space data is affected by $T_{2}^{*}$ weighting increases with increasing total read-out time. Consequently longer total read-out times in SE result in decreased spatial specificity (Goense and Logothetis, 2006) and are expected to have a wider point-spread function (though still narrower than GE).

Other $T_{2}$ based functional imaging methods such as GRASE (Oshio and Feinberg, 1991) and 3D-GRASE (Feinberg et al., 2008) are expected to have similar spatial specificity as SE. Whether their PSFs are slightly wider or narrower will mainly depend on the $T_{2}^{*}$ weighting component associated with such methods (i.e. echo train lengths of gradient recalled echoes employed in between successive $180^{\circ}$ pulses), in addition to their $T_{2}$ component. In fact, Kem- 
per et al. (2015) have reported that 3D-GRASE had a smaller bias towards pial surface veins owing to the smaller $T_{2}^{*}$ contribution when a reduced field of view is employed in zoomed 3D-GRASE compared to the longer in-plane echotrain of 2D-SE EPI.

Although we found a wider PSF for GE BOLD than for SE BOLD FMRI, the difference was relatively small $(1.02 \mathrm{~mm}$ for GE BOLD, $0.82 \mathrm{~mm}$ for SE BOLD). We believe that this is due to the fact that the influence of larger blood vessels can be reduced by using a differential imaging paradigm, even when using 7T GE BOLD fMRI. Consequently, both GE and SE BOLD imaging techniques seem capable of resolving cortical columns when applying differential imaging analysis.

However, GE maps are more susceptible to confounds introduced in voxels containing blood vessels which may not be fully suppressed in differential imaging. Therefore, obtaining results of high spatial specificity using GE depends on the region of interest and on methods to mask out blood vessels.

SE is less susceptible to large-vessel confounds, that may not be suppressed by differential imaging. The response amplitude of SE is lower than that of GE. However, for imaging of highly granular structures such as ODC's at such high resolutions, the differential contrast is similar for GE and SE fMRI. Overall, we believe that SE is the method of choice for mapping finer structures, especially when relying on single-condition analysis. However, which data acquisition method is optimal depends on the goal of the study and the spatial scale of the neuronal architecture under investigation.

\section{The application of probabilistic models of cortical columns and MR imaging}

We have extended our quantitative model for imaging ODCs to a probabilistic generative model and used it to infer the PSF widths by means of MCMC sampling.

A critical component to the successful application of MCMC to our model is the Hamiltonian Monte Carlo (HMC) algorithm (Duane et al., 1987), which makes use of the gradient of the model posterior probability. Importantly, we were able to derive an efficient way to compute this gradient (Appendices $\mathrm{C}$ and D). HMC has the advantage of very efficiently exploring the parameter space. However, for high-dimensional problems such as ours, every step may take a long time because the gradient components for all variables need to be computed. Because of the specific form of the computations in our model (convolutions and a point-wise non-linearity), it was possible to compute the gradient efficiently as a combination of convolutions and point-wise nonlinearities as well. In principle, such efficient computation should be possible for a wide range of similar models, making HMC a powerful method for fitting such models.

We believe that the novel approach we introduce to the field of imaging cortical columns, of fitting a model of imaging columns to corresponding measured data, will be useful beyond our current study. For example, when imaging an unknown columnar structure, questions about its organization (e.g. isotropy, spatial frequency, irregularity) can be addressed via inference on model parameters. 


\section{Conclusion}

We have quantified the BOLD PSF in human subjects relative to neuronal activity, avoiding the confounding effects of scatter and size of visual receptive fields which were not eliminated in previous estimations (Engel et al., 1997; Parkes et al., 2005; Shmuel et al., 2007). As a result, our BOLD PSF estimates characterize the spatial specificity when employing imaging of fine scale cortical organizations such as cortical columns. Previous studies have shown that BOLD fMRI at $4 \mathrm{~T}$ and $7 \mathrm{~T}$ is capable of resolving cortical columns on the submillimeter scale when differential analysis is employed (Cheng et al., 2001; Menon and Goodyear, 1999; Yacoub et al., 2008; 2007; Zimmermann et al., 2011). Our results provide a quantitative basis for this resolvability and facilitate planning and interpretation of high-resolution fMRI studies of fine scale cortical organizations.

\section{References}

Adams, D.L., Sincich, L.C., Horton, J.C., 2007. Complete pattern of ocular dominance columns in human primary visual cortex. J Neurosci 27, 10391-10403. doi:10.1523/JNEUROSCI.2923-07.2007

Aquino, K.M., Robinson, P.A., Schira, M.M., Breakspear, M., 2014. Deconvolution of neural dynamics from fMRI data using a spatiotemporal hemodynamic response function. Neuroimage 94, 203-215. doi:10.1016/j.neuroimage.2014.03.001

Aquino, K.M., Schira, M.M., Robinson, P.A., Drysdale, P.M., Breakspear, M., 2012. Hemodynamic traveling waves in human visual cortex. PLoS Comput Biol 8, e1002435. doi:10.1371/journal.pcbi.1002435

Bandettini, P.A., Wong, E.C., Hinks, R.S., Tikofsky, R.S., Hyde, J.S., 1992. Time course EPI of human brain function during task activation. Magn Reson Med 25, 390-397.

Berens, P., Keliris, G.A., Ecker, A.S., Logothetis, N.K., Tolias, A.S., 2008. Comparing the feature selectivity of the gamma-band of the local field potential and the underlying spiking activity in primate visual cortex. Front Syst Neurosci 2, 2. doi:10.3389/neuro.06.002.2008

Chaimow, D., Shmuel, A., 2016. A more accurate account of the effect of k-space sampling and signal decay on the effective spatial resolution in functional MRI. bioRxiv.

Chaimow, D., Yacoub, E., Ugurbil, K., Shmuel, A., 2011. Modeling and analysis of mechanisms underlying fMRI-based decoding of information conveyed in cortical columns. Neuroimage 56, 627-642. doi:10.1016/j.neuroimage.2010.09.037

Cheng, K., Waggoner, R., Tanaka, K., 2001. Human ocular dominance columns as revealed by high-field functional magnetic resonance imaging. Neuron 32, 359-374.

Cox, R.W., Jesmanowicz, A., 1999. Real-time 3D image registration for functional MRI. Magn Reson Med 42, 1014-1018.

De Martino, F., Moerel, M., Ugurbil, K., Goebel, R., Yacoub, E., Formisano, E., 2015. Frequency preference and attention effects across cortical depths in the human primary auditory cortex. Proceedings of the National Academy of Sciences 112, 16036-16041. doi:10.1073/pnas.1507552112

Duane, S., Kennedy, A.D., Pendleton, B.J., Roweth, D., 1987. Hybrid Monte Carlo. Physics letters B.

Duong, T.Q., Kim, D.S., Ugurbil, K., Kim, S.G., 2001. Localized cerebral blood flow 
response at submillimeter columnar resolution. Proceedings of the National Academy of Sciences 98, 10904-10909. doi:10.1073/pnas.191101098

Duong, T.Q., Yacoub, E., Adriany, G., Hu, X., Ugurbil, K., Kim, S.-G., 2003. Microvascular BOLD contribution at 4 and $7 \mathrm{~T}$ in the human brain: Gradient-echo and spin-echo fMRI with suppression of blood effects. Magn Reson Med 49, 10191027.

Duvernoy, H.M., Delon, S., Vannson, J.L., 1981. Cortical blood vessels of the human brain. Brain Res Bull 7, 519-579.

Engel, S., Glover, G., Wandell, B.A., 1997. Retinotopic organization in human visual cortex and the spatial precision of functional MRI. Cereb Cortex 7, 181-192.

Feinberg, D.A., Harel, N., Ramanna, S., Ugurbil, K., Yacoub, E., 2008. Sub-millimeter Single-shot 3D GRASE with Inner Volume Selection for T2 weighted fMRI applications at 7 Tesla. Proc. Intl. Soc. Mag. Reson. Med. 16, 1-1.

Fracasso, A., Petridou, N., Dumoulin, S.O., 2016. Systematic variation of population receptive field properties across cortical depth in human visual cortex. Neuroimage 139, 427-438. doi: 10.1016/j.neuroimage.2016.06.048.

Geweke, J., 1991. Evaluating the accuracy of sampling-based approaches to the calculation of posterior moments.

Goense, J.B.M., Logothetis, N.K., 2006. Laminar specificity in monkey V1 using highresolution SE-fMRI. Magn Reson Imaging 24, 381-392.

Goodyear, B.G., Menon, R.S., 2001. Brief visual stimulation allows mapping of ocular dominance in visual cortex using fMRI. Hum Brain Mapp 14, 210-217.

Haacke, M.E., Brown, R.W., Thompson, M.R., 1999. Magnetic resonance imaging: physical principles and sequence design 914.

Horton, J.C., Hoyt, W.F., 1991. The representation of the visual field in human striate cortex. A revision of the classic Holmes map. Arch Ophthalmol 109, 816-824.

Hubel, D., Wiesel, T., 1968. Receptive fields and functional architecture of monkey striate cortex. J Physiol (Lond) 195, 215-243.

Hubel, D.H., Wiesel, T.N., 1974. Uniformity of monkey striate cortex: a parallel relationship between field size, scatter, and magnification factor. J Comp Neurol 158, 295-305. doi:10.1002/cne.901580305

Jenkinson, M., Bannister, P., Brady, M., Smith, S., 2002. Improved optimization for the robust and accurate linear registration and motion correction of brain images. Neuroimage 17, 825-841.

Jenkinson, M., Smith, S., 2001. A global optimisation method for robust affine registration of brain images. Medical Image Analysis 5, 143-156.

Jochimsen, T.H., Norris, D.G., Mildner, T., Möller, H.E., 2004. Quantifying the intraand extravascular contributions to spin-echo fMRI at 3 T. Magn Reson Med 52, 724-732. doi:10.1002/mrm.20221

Kemper, V.G., De Martino, F., Vu, A.T., Poser, B.A., Feinberg, D.A., Goebel, R., Yacoub, E., 2015. Sub-millimeter T2 weighted fMRI at $7 \mathrm{~T}$ : comparison of 3D-GRASE and 2D SE-EPI. Front Neurosci 9, 163. doi:10.3389/fnins.2015.00163

Kriegeskorte, N., Cusack, R., Bandettini, P., 2010. How does an fMRI voxel sample the neuronal activity pattern: compact-kernel or complex spatiotemporal filter? Neuroimage 49, 1965-1976. doi:10.1016/j.neuroimage.2009.09.059

Kwong, K.K., Belliveau, J.W., Chesler, D.A., Goldberg, I.E., Weisskoff, R.M., Poncelet, B.P., Kennedy, D.N., Hoppel, B.E., Cohen, M.S., Turner, R., 1992. Dynamic magnetic resonance imaging of human brain activity during primary sensory stimulation. Proceedings of the National Academy of Sciences 89, 5675-5679.

Lagarias, J.C., Reeds, J.A., Wright, M.H., Wright, P.E., 1998. Convergence Properties of the Nelder--Mead Simplex Method in Low Dimensions. SIAM J. Optim. 9, 112- 
147. doi:10.1137/S1052623496303470

Leys, C., Ley, C., Klein, O., Bernard, P., Licata, L., 2013. Detecting outliers: Do not use standard deviation around the mean, use absolute deviation around the median. Journal of Experimental Social Psychology 49, 764-766. doi:10.1016/j.jesp.2013.03.013

Menon, R.S., Goodyear, B.G., 1999. Submillimeter functional localization in human striate cortex using BOLD contrast at 4 Tesla: implications for the vascular pointspread function. Magn Reson Med 41, 230-235.

Menon, R.S., Ogawa, S., Strupp, J., Ugurbil, K., 1997. Ocular dominance in human V1 demonstrated by functional magnetic resonance imaging. J Neurophysiol 77, 2780-2787.

Muckli, L., De Martino, F., Vizioli, L., Petro, L.S., Smith, F.W., 2015. Contextual Feedback to Superficial Layers of V1. Curr Biol.

Neal, R., 2011. MCMC using Hamiltonian dynamics, in:. Handbook of Markov Chain Monte Carlo, pp. 113-162.

Neal, R.M., 1993. Probabilistic inference using Markov chain Monte Carlo methods.

Noll, D.C., Nishimura, D.G., Macovski, A., 1991. Homodyne detection in magnetic resonance imaging. Ieee Transactions on Medical Imaging 10, 154-163. doi:10.1109/42.79473

Ogawa, S., Lee, T.M., Kay, A.R., Tank, D.W., 1990. Brain magnetic resonance imaging with contrast dependent on blood oxygenation. Proceedings of the National Academy of Sciences 87, 9868-9872.

Ogawa, S., Tank, D.W., Menon, R., Ellermann, J.M., Kim, S.G., Merkle, H., Ugurbil, K., 1992. Intrinsic signal changes accompanying sensory stimulation: functional brain mapping with magnetic resonance imaging. Proceedings of the National Academy of Sciences 89, 5951-5955.

Olman, C.A., Harel, N., Feinberg, D.A., He, S., Zhang, P., Ugurbil, K., Yacoub, E., 2012. Layer-specific fMRI reflects different neuronal computations at different depths in human V1. PLoS ONE 7, e32536-9. doi:10.1371/journal.pone.0032536

Oshio, K., Feinberg, D.A., 1991. GRASE (Gradient- and spin-echo) imaging: a novel fast MRI technique. Magn Reson Med 20, 344-349.

Parkes, L.M., Schwarzbach, J.V., Bouts, A.A., Deckers, R.H.R., Pullens, P., Kerskens, C.M., Norris, D.G., 2005. Quantifying the spatial resolution of the gradient echo and spin echo BOLD response at 3 Tesla. Magn Reson Med 54, 1465-1472. doi:10.1002/mrm.20712

Pfeuffer, J., van de Moortele, P.F., Ugurbil, K., Hu, X., Glover, G., 2002. Correction of physiologically induced global off resonance effects in dynamic echo-planar and spiral functional imaging. Magn Reson Med 47, 344-353.

Polimeni, J.R., Fischl, B., Greve, D.N., Wald, L.L., 2010. Laminar analysis of 7T BOLD using an imposed spatial activation pattern in human V1. Neuroimage 52, 1334 1346. doi:10.1016/j.neuroimage.2010.05.005

Rojer, A., Schwartz, E., 1990. Cat and monkey cortical columnar patterns modeled by bandpass-filtered 2D white noise. Biol Cybern 62, 381-391.

Shmuel, A., Chaimow, D., Raddatz, G., Ugurbil, K., Yacoub, E., 2010. Mechanisms underlying decoding at $7 \mathrm{~T}$ : ocular dominance columns, broad structures, and macroscopic blood vessels in V1 convey information on the stimulated eye. Neuroimage 49, 1957-1964. doi:10.1016/j.neuroimage.2009.08.040

Shmuel, A., Yacoub, E., Chaimow, D., Logothetis, N.K., Ugurbil, K., 2007. Spatiotemporal point-spread function of fMRI signal in human gray matter at 7 Tesla. Neuroimage 35, 539-552. doi:10.1016/j.neuroimage.2006.12.030

Turner, R., 2002. How much cortex can a vein drain? Downstream dilution of activa- 
tion-related cerebral blood oxygenation changes. Neuroimage 16, 1062-1067. doi:10.1006/nimg.2002.1082

Uludağ, K., Müller-Bierl, B., Ugurbil, K., 2009. An integrative model for neuronal activity-induced signal changes for gradient and spin echo functional imaging. Neuroimage 48, 150-165. doi:10.1016/j.neuroimage.2009.05.051

Vazquez, A.L., Fukuda, M., Crowley, J.C., Kim, S.-G., 2014. Neural and hemodynamic responses elicited by forelimb- and photo-stimulation in channelrhodopsin-2 mice: insights into the hemodynamic point spread function. Cereb Cortex 24, 2908-2919. doi:10.1093/cercor/bht147

Weber, B., Keller, A., Reichold, J., Logothetis, N.K., 2008. The Microvascular System of the Striate and Extrastriate Visual Cortex of the Macaque. Cereb Cortex 18, 2318-2330. doi:10.1093/cercor/bhm259

Xing, D., Yeh, C.-I., Shapley, R.M., 2009. Spatial spread of the local field potential and its laminar variation in visual cortex. J Neurosci 29, 11540-11549. doi:10.1523/JNEUROSCI.2573-09.2009

Yacoub, E., Duong, T.Q., van de Moortele, P.F., Lindquist, M., Adriany, G., Kim, S.-G., Ugurbil, K., Hu, X., 2003. Spin-echo fMRI in humans using high spatial resolutions and high magnetic fields. Magn Reson Med 49, 655-664.

Yacoub, E., Harel, N., Ugurbil, K., 2008. High-field fMRI unveils orientation columns in humans. Proceedings of the National Academy of Sciences 105, 10607-10612. doi:10.1073/pnas.0804110105

Yacoub, E., Shmuel, A., Logothetis, N.K., Ugurbil, K., 2007. Robust detection of ocular dominance columns in humans using Hahn Spin Echo BOLD functional MRI at 7 Tesla. Neuroimage 37, 1161-1177.

Yacoub, E., Shmuel, A., Pfeuffer, J., van de Moortele, P.F., Adriany, G., Andersen, P., Vaughan, J.T., Merkle, H., Ugurbil, K., Hu, X., 2001. Imaging brain function in humans at 7 Tesla. Magn Reson Med 45, 588-594.

Zimmermann, J., Goebel, R., De Martino, F., van de Moortele, P.-F., Feinberg, D., Adriany, G., Chaimow, D., Shmuel, A., Ugurbil, K., Yacoub, E., 2011. Mapping the organization of axis of motion selective features in human area MT using highfield fMRI. PLoS ONE 6, e28716. doi:10.1371/journal.pone.0028716. 


\section{Appendix A. Model of imaging ocular dominance columns}

\section{Preliminaries}

Simulations before MRI sampling were carried out on a grid of size $N_{1}^{\text {sim }} \times N_{2}^{\text {sim }}$. Simulations of MRI data were of size $N_{1}^{M R I} \times N_{2}^{M R I}$. We use $i, j$ and $k, l$ as indices of 2-dimensional image and spatial frequency space, respectively. Furthermore, $r(k, l)$ is the absolute spatial frequency and $\phi(k, l)$ the orientation that the point with indices $k, l$ represents. The two-dimensional discrete Fourier transform and its inverse (D.3 and D.4) are denoted as $\mathrm{dft} 2$ and idft2.

\section{Overview over model computations}

The ocular dominance columns (ODCs) imaging model can be described as a function

$$
f(q)=\left(f_{G E}(q), f_{S E}(q)\right),
$$

that takes the the list of model parameter values $q=\left(\left(n_{i, j}\right), \rho, \delta, \epsilon, \theta, \omega, f w h m_{G E}, f w h m_{S E}\right)$ and the fixed parameters $\beta_{G E}$ and $\beta_{S E}$ (see Table 1) as input and generates differential fMRI maps of ODC $f_{G E}(q)=\left(m r i_{i, j}^{G E}\right)$ and $f_{S E}(q)=\left(m r i_{i, j}^{S E}\right)$ in a number of steps:

$$
\begin{aligned}
& \left(\widetilde{o d c}_{i, j}\right)=\operatorname{idft} 2\left[\left(\operatorname{dft} 2\left[\left(n_{i, j}\right)\right]_{k, l} \cdot F_{k, l}^{O D C}(\rho, \delta, \epsilon, \theta)\right)\right], \\
& o d c_{i, j}=s\left(\widetilde{o d c}_{i, j}, \omega\right), \\
& \left(\operatorname{bold}_{i, j}^{G E}\right)=\operatorname{idft} 2\left[\left(\operatorname{dft} 2\left[\left(\operatorname{odc}_{i, j}\right)\right]_{k, l} \cdot \operatorname{MTF}_{k, l}\left(f w h m_{G E}, \beta_{G E}\right)\right)\right] \text {, } \\
& \left(\operatorname{bold}_{i, j}^{S E}\right)=\operatorname{idft} 2\left[\left(\operatorname{dft} 2\left[\left(o d c_{i, j}\right)\right]_{k, l} \cdot M T F_{k, l}\left(f w h m_{S E}, \beta_{S E}\right)\right)\right] \text {, } \\
& \left(m r i i_{i, j}^{G E}\right)=\operatorname{idft} 2\left[\left(\operatorname{dft} 2\left[\left(\operatorname{bold}_{i, j}^{G E}\right)\right]_{\substack{k=I_{1}^{M R I}(1), \ldots, I_{1}^{M R I}\left(N_{1}^{M R I}\right), l=I_{2}^{M R I}(1), \ldots, I_{2}^{M R I}\left(N_{2}^{M R I}\right)}}\right)\right] \frac{N_{1}^{M R I} N_{2}^{M R I}}{N_{1}^{s i m} N_{2}^{s i m}}, \\
& \left(m r i i_{i, j}^{S E}\right)=\operatorname{idft} 2\left[\left(\operatorname{dft} 2\left[\left(\operatorname{bold}_{i, j}^{S E}\right)\right]_{\substack{k=I_{1}^{M R I}(1), \ldots, I_{1}^{M R I}\left(N_{1}^{M R I}\right), l=I_{2}^{M R I}(1), \ldots, I_{2}^{M R I}\left(N_{2}^{M R I}\right)}}\right)\right] \frac{N_{1}^{M R I} N_{2}^{M R I}}{N_{1}^{s i m} N_{2}^{s i m}} .
\end{aligned}
$$

(A.1): The ODC pattern (odc $c_{i, j}$ ) was modeled by filtering two-dimensional Gaussian white noise $\left(n_{i, j}\right)$ using a non-isotropic filter $\left(F_{k, l}^{O D C}\right)$ and followed by (A.2) a point-wise sigmoidal non-linearity $s$ that controlled the smoothness of transitions between left and right eye preferrence columns. (A.3 and A.4): The BOLD response was modeled as a convolution with a Gaussian point-spread function. It was implemented as multiplication in spatial frequency space with its Fourier transform the modulation transfer function $\left(M T F_{k, l}\right)$. (A.5 and A.6): MRI sampling was simulated by restricting the spatial frequency space representation to its central part (indices given by index functions $I_{1}^{M R I}$ and $I_{2}^{M R I}$ ) in accordance with the voxel size. The last factor corrects for the reduction in scale caused by applying idft2 to the reduced grid size.

We can combine operations A.3 and A.5, as well as A.4 and A.6:

$$
\begin{aligned}
& \left(m r i_{i, j}^{G E}\right)=\operatorname{idft} 2\left[\left(\operatorname{dft} 2\left[\left(\operatorname{odc}_{i, j}^{G E}\right)\right]_{\substack{k=I_{1}^{M R I}(1), \ldots . I_{1}^{M R I}\left(N_{1}^{M R I}\right) \\
l=I_{2}^{M R I}(1), \ldots, I_{2}^{M R I}\left(N_{2}^{M R I}\right)}} \cdot F_{k, l}^{B O L D}\left(f w h m_{G E}, \beta_{G E}\right)\right)\right], \\
& \left(m r i_{i, j}^{S E E}\right)=\operatorname{idft} 2\left[\left(\operatorname{dft} 2\left[\left(\operatorname{odc}_{i, j}^{S E}\right)\right]_{\substack{k=I_{1}^{M R I}(1), \ldots, I_{1}^{M R I}\left(N_{1}^{M R I}\right) \\
l=I_{2}^{M R I}(1), \ldots, I_{2}^{M R I}\left(N_{2}^{M R I}\right)}} \cdot F_{k, l}^{B O L D}\left(f^{M R h} m_{S E}, \beta_{S E}\right)\right)\right],
\end{aligned}
$$

where $\left(F_{k, l}^{B R I}\right)$ is of size $N_{1}^{M R I} \times N_{2}^{M R I}$ with:

$$
F_{k, l}^{B O L D}(f w h m, \beta)=\operatorname{MTF}_{I_{1}^{M R I}(k), I_{2}^{M R I}(l)}(f w h m, \beta) \frac{N_{1}^{M R I} N_{2}^{M R I}}{N_{1}^{s i m} N_{2}^{s i m}}
$$


ODC filter

An unnormalized ODC filter $\tilde{F}_{k, l}^{O D C}$ was defined in spatial frequency space as the product of radial and angular components:

$$
\tilde{F}_{k, l}^{O D C}=\tilde{F}_{k, l}^{O D C_{\text {rad }}} \cdot \tilde{F}_{k, l}^{O D C_{\text {ang }}}
$$

The radial component $\tilde{F}_{k, l}^{O D C_{\text {rad }}}$ is the sum of two Gaussian functions centered on $+\rho$ and $-\rho$, where $\rho$ is the main spatial frequency of the pattern.

$$
\begin{aligned}
& \tilde{F}_{k, l}^{O D C_{r a d}}=\tilde{F}_{k, l}^{O D C_{r a d}^{+}}+\tilde{F}_{k, l}^{O D C_{r a d}^{-}}, \\
& \tilde{F}_{k, l}^{O D C_{r a d}^{+}}=e^{-\frac{(r(k, l)-\rho)^{2}}{2 \delta^{2}}}, \\
& \tilde{F}_{k, l}^{O D C_{r a d}^{-}}=e^{-\frac{(r(k, l)-(-\rho))^{2}}{2 \delta^{2}}} .
\end{aligned}
$$

The angular component is the sum of two von Mises distribution functions centered on $+\theta$ and $-\theta$, where $\theta$ is the orientation of the pattern:

$$
\begin{aligned}
& \tilde{F}_{k, l}^{O D C_{a n g}}=\tilde{F}_{k, l}^{O D C_{a n g}^{1}}+\tilde{F}_{k, l}^{O D C_{a n g}^{2}}, \\
& \tilde{F}_{k, l}^{O D C_{a n g}^{1}}=e^{\frac{\cos (\phi(k, l)-\theta)}{\epsilon^{2}}}, \\
& \tilde{F}_{k, l}^{O D C_{a n g}^{2}}=e^{\frac{\cos (\phi(k, l)-(\theta+\pi))}{\epsilon^{2}}}=e^{\frac{-\cos (\phi(k, l)-\theta)}{\epsilon^{2}}} .
\end{aligned}
$$

In order for the filter output to have the same variance as the input (independent of filter parameter values) we normalized the filter:

$$
F_{k, l}^{O D C}=\frac{\tilde{F}_{k, l}^{O D C}}{C^{O D C}}
$$

where

$$
C^{O D C}=\sqrt{\frac{\sum_{k, l=1}^{N_{1}^{s i m} \times N_{2}^{s i m}}\left(\tilde{F}_{k, l}^{O D C}\right)^{2}}{N_{1}^{s i m} N_{2}^{s i m}}} .
$$

Sigmoidal non-linearity

The point-wise sigmoidal non-linearity $s(x, \omega)$ was defined as:

$$
s(x, \omega)=2\left(\tilde{s}\left(\frac{x}{\omega}\right)-0.5\right),
$$

with the standard sigmoidal function defined as:

BOLD response

$$
\tilde{s}(x)=\frac{1}{1+e^{-x}} .
$$

The BOLD response modulation transfer function was defined as

$$
\operatorname{MTF}_{k, l}(f w h m, \beta)=\beta \cdot e^{-2 \pi^{2} \sigma_{\text {BOLD }}^{2} \cdot r(k, l)^{2}},
$$

with:

$$
\sigma_{B O L D}=\frac{f w h m}{2 \sqrt{2 \log 2}} \approx \frac{f w h m}{2.35} .
$$

It is the Fourier transform of a Gaussian point-spread function with a full-width at half-maximum of fwhm. It is scaled such that a spatially extended neuronal response of 1 results in a BOLD response of amplitude $\beta$. 


\section{MRI sampling}

MRI sampling was simulated by restricting the spatial frequency representation according to the following index functions:

$$
\begin{aligned}
& I_{1}^{M R I}(k)=\left\{\begin{array}{ll}
k & \text { if } k \leq \frac{N_{1}^{M R I}}{2} \\
k-N_{1}^{M R I}+N_{1}^{s i m} & \text { if } k>\frac{N_{1}^{M R I}}{2}
\end{array},\right. \\
& I_{2}^{M R I}(l)=\left\{\begin{array}{ll}
l & \text { if } l \leq \frac{N_{2}^{M R I}}{2} \\
l-N_{2}^{M R I}+N_{2}^{\text {sim }} & \text { if } l>\frac{N_{2}^{\text {MRI }}}{2}
\end{array} .\right.
\end{aligned}
$$

\section{Appendix B. Posterior probability and potential energy}

\section{Posterior probability}

Let $\mathscr{D}=\left\{\left(\right.\right.$ data $\left._{i, j}^{G E}\right),\left(\right.$ data $\left.\left._{i, j}^{S E}\right)\right\}$ be the data of differential fMRI maps imaged using GE and SE BOLD fMRI, respectively. The likelihood - the probability to observe the data $\mathscr{D}$ given a specific set of model parameter values $q$ is:

$$
P[\mathscr{D} \mid q]=P\left[\left(\operatorname{data}_{i, j}^{G E}\right)=f_{G E}(q)+\left(v_{i, j}^{G E}\right),\left(\operatorname{data}_{i, j}^{S E}\right)=f_{S E}(q)+\left(v_{i, j}^{S E}\right) \mid q\right],
$$

where $\left(v_{i, j}^{G E}\right)$ and $\left(v_{i, j}^{S E}\right)$ are patterns of measurement noise.

We assume the measurement noise to be independent between voxels and imaging modalities and to be distributed normally with (estimated) variances $\hat{\sigma}_{G E}^{2}$ and $\hat{\sigma}_{S E}^{2}$. Furthermore we define $\left(d_{i, j}^{G E}\right)=$ $\left(\right.$ data $\left._{i, j}^{G E}\right)-\left(m r_{i, j}^{G E}\right)$ and $\left(d_{i, j}^{S E}\right)=\left(\right.$ data $\left._{i, j}^{S E}\right)-\left(m r_{i, j}^{S E}\right)$ to be the patterns of deviations of the data from the model prediction. The likelihood can then be expressed as:

$$
P[\mathscr{D} \mid q]=\frac{1}{\left(2 \pi \hat{\sigma}_{G E} \hat{\sigma}_{S E}\right)^{N_{1}^{M R I} N_{2}^{M R I}}} \prod_{i, j=1}^{N_{1}^{M R I} \times N_{2}^{M R I}} e^{-\frac{\left(d_{i, j}^{G E}\right)^{2}}{2 \tilde{\sigma}_{G E}^{2}}-\frac{\left(d_{i, j}\right)^{2}}{2 \tilde{\sigma}_{S E}^{2}}}
$$

From this result we can calculate the posterior probability of parameters $q$ given the data $\mathscr{D}$ :

$$
\begin{aligned}
P[q \mid \mathscr{D}] & =\frac{P[\mathscr{D} \mid q] P[q]}{P[\mathscr{D}]} \\
& =A \cdot P[q] \cdot \prod_{i, j=1}^{N_{1}^{M R I} \times N_{2}^{M R I}} e^{-\frac{\left(d_{i, j}^{G E}\right)^{2}}{2 \tilde{\sigma}_{G E}^{2}}-\frac{\left(d_{i, j}^{S E}\right)^{2}}{2 \tilde{\sigma}_{S E}^{2}}}
\end{aligned}
$$

where $A=\frac{1}{P[\mathscr{D}] \cdot\left(2 \pi \hat{\sigma}_{G E} \hat{\sigma}_{S E}\right)^{N_{1}^{M R I} N_{2}^{M R I}}}$ is a constant factor that is independent of $q$ and $P[q]$ is the prior probability over parameters. 
Prior probability

The prior probability over all parameters $q$ was defined as:

$$
P[q]=\left\{\begin{array}{ll}
0 & \text { if } \omega<0 \\
& \text { or } f w h m_{G E}<0 \\
& \text { or } f_{h h m_{S E}<0} \\
P\left[\left(n_{i, j}\right)\right] \cdot P[\rho] \cdot P[\delta] \cdot P[\epsilon] \cdot P[\omega] & \text { otherwise }
\end{array},\right.
$$

with individual parameter priors were defined as:

$$
\begin{aligned}
& P\left[\left(n_{i, j}\right)\right] \propto e^{-\frac{\sum_{i, j=1}^{N_{1}^{s i m} \times N_{2}^{s i m}} n_{i, j}^{2}}{2}}, \\
& P[\rho] \propto\left\{\begin{array}{ll}
0 & \text { if }\left|\rho-\mu_{\rho}\right|>2 \sigma_{\rho} \\
e^{-\frac{\left(\rho-\mu_{\rho}\right)^{2}}{2 \sigma_{\rho}^{2}}} & \text { otherwise }
\end{array},\right. \\
& P[\delta] \propto\left\{\begin{array}{ll}
0 & \text { if } \delta<0 \\
e^{-\frac{\left(\delta-\mu_{\delta}\right)^{2}}{2 \sigma_{\delta}^{2}}} & \text { otherwise }
\end{array},\right. \\
& P[\epsilon] \propto\left\{\begin{array}{ll}
0 & \text { if } \epsilon<0 \\
e^{-\frac{\left(\epsilon-\mu_{\epsilon}\right)^{2}}{2 \sigma_{\epsilon}^{2}}} & \text { otherwise }
\end{array},\right. \\
& P[\omega] \propto \begin{cases}0 & \text { if } \omega<\omega_{\min } \\
0 & \text { if } \omega>\omega_{\max } \\
1 & \text { otherwise }\end{cases}
\end{aligned}
$$

where $\mu_{\rho}, \mu_{\delta}, \mu_{\epsilon}$ and $\sigma_{\rho}, \sigma_{\delta}, \sigma_{\epsilon}$ are the means and standard deviations of the ODC priors that were estimated from cytochrome oxidase data and $\omega_{\min }$ and $\omega_{\max }$ are lower and upper limits for $\omega$ that were set based on results from the neurophysiological literature.

\section{Potential energy}

The potential energy of a state of parameter values $q$ is its negative log-posterior probability plus an arbitrary constant $C$ :

$$
E(q)=-\log P[q \mid \mathscr{D}]+C .
$$

We apply B.1 and express the energy as the sum of three parts:

$$
=E_{G E}(q)+E_{S E}(q)+E(q)_{\text {prior }},
$$

with:

$$
\begin{aligned}
& E_{G E}(q)=\sum_{i, j=1}^{N_{1}^{M R I} \times N_{2}^{M R I}} \frac{\left(d_{i, j}^{G E}\right)^{2}}{2 \hat{\sigma}_{G E}^{2}}, \\
& E_{S E}(q)=\sum_{i, j=1}^{N_{1}^{M R I} \times N_{2}^{M R I}} \frac{\left(d_{i, j}^{S E}\right)^{2}}{2 \hat{\sigma}_{S E}^{2}}
\end{aligned}
$$


and

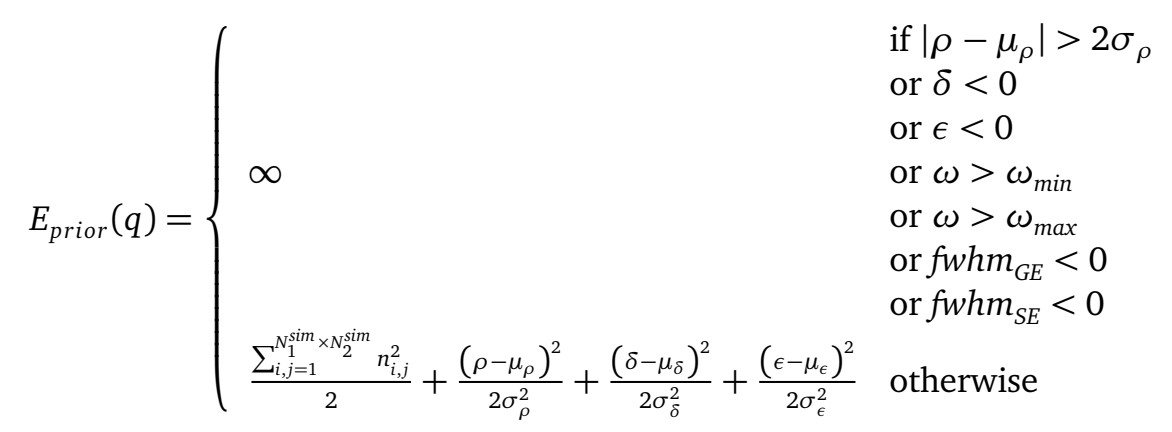

\section{Appendix C. Potential energy gradient}

We start by deriving derivatives for functions used by the model.

Derivatives of the ODC filter

The derivatives of the unnormalized radial and angular filter component parts with respect to their prarameters are:

$$
\begin{gathered}
\frac{\partial \tilde{F}_{k, l}^{O D C_{r a d}^{+}}}{\partial \rho}=\tilde{F}_{k, l}^{O D C_{r a d}^{+}} \cdot \frac{r(k, l)-\rho}{\delta^{2}}, \\
\frac{\partial \tilde{F}_{k, l}^{O D C_{r a d}^{-}}}{\partial \rho}=\tilde{F}_{k, l}^{O D C_{r a d}^{-}} \cdot-\frac{r(k, l)-(-\rho)}{\delta^{2}}, \\
\frac{\partial \tilde{F}_{k, l}^{O D C_{r a d}^{+}}}{\partial \delta}=\tilde{F}_{k, l}^{O D C_{r a d}^{+}} \cdot \frac{(r(k, l)-\rho)^{2}}{\delta^{3}}, \\
\frac{\partial \tilde{F}_{k, l}^{O D C_{r a d}^{-}}}{\partial \delta}=\tilde{F}_{k, l}^{O D C_{r a d}^{-}} \cdot \frac{(r(k, l)-(-\rho))^{2}}{\delta^{3}}, \\
\frac{\partial \tilde{F}_{k, l}^{O D C_{a n g}^{1}}}{\partial \theta}=\tilde{F}_{k, l}^{O D C_{a n g}^{1}} \cdot \frac{\sin (\phi(k, l)-\theta)}{\epsilon^{2}}, \\
\frac{\partial \tilde{F}_{k, l}^{O D C_{a n g}^{2}}}{\partial \theta}=\tilde{F}_{k, l}^{O D C_{a n g}^{2}} \cdot-\frac{\sin (\phi(k, l)-\theta)}{\epsilon^{2}} \\
\frac{\partial \tilde{F}_{k, l}^{O D C_{a n g}^{1}}}{\partial \epsilon}=\tilde{F}_{k, l}^{O D C_{a n g}^{1}} \cdot-2 \frac{\cos (\phi(k, l)-\theta)}{\epsilon^{3}}
\end{gathered}
$$


We combine these derivatives to form derivatives of the full radial and angular components:

$$
\begin{aligned}
& \frac{\partial \tilde{F}_{k, l}^{O D C_{r a d}}}{\partial \rho}=\frac{\partial \tilde{F}_{k, l}^{O D C_{r a d}^{+}}}{\partial \rho}+\frac{\partial \tilde{F}_{k, l}^{O D C_{r a d}^{-}}}{\partial \rho,} \\
& \frac{\partial \tilde{F}_{k, l}^{O D C_{r a d}}}{\partial \delta}=\frac{\partial \tilde{F}_{k, l}^{O D C_{r a d}^{+}}}{\partial \delta}+\frac{\partial \tilde{F}_{k, l}^{O D C_{r a d}^{-}}}{\partial \delta}, \\
& \frac{\partial \tilde{F}_{k, l}^{O D C_{a n g}}}{\partial \theta}=\frac{\partial \tilde{F}_{k, l}^{O D C_{a n g}^{1}}}{\partial \theta}+\frac{\partial \tilde{F}_{k, l}^{O D C_{a n g}^{2}}}{\partial \theta}, \\
& \frac{\partial \tilde{F}_{k, l}^{O D C_{a n g}}}{\partial \epsilon}=\frac{\partial \tilde{F}_{k, l}^{O D C_{a n g}^{1}}}{\partial \epsilon}+\frac{\partial \tilde{F}_{k, l}^{O D C_{a n g}^{2}}}{\partial \epsilon},
\end{aligned}
$$

and in turn to form the derivatives of the full unnormalized filter:

$$
\begin{aligned}
& \frac{\partial \tilde{F}_{k, l}^{O D C}}{\partial \rho}=\frac{\partial \tilde{F}_{k, l}^{O D C_{r a d}}}{\partial \rho} \cdot \tilde{F}_{k, l}^{O D C_{a n g}}, \\
& \frac{\partial \tilde{F}_{k, l}^{O D C}}{\partial \delta}=\frac{\partial \tilde{F}_{k, l}^{O D C_{r a d}}}{\partial \delta} \cdot \tilde{F}_{k, l}^{O D C_{a n g}}, \\
& \frac{\partial \tilde{F}_{k, l}^{O D C}}{\partial \theta}=\tilde{F}_{k, l}^{O D C_{r a d}} \cdot \frac{\partial \tilde{F}_{k, l}^{O D C_{a n g}}}{\partial \theta}, \\
& \frac{\partial \tilde{F}_{k, l}^{O D C}}{\partial \epsilon}=\tilde{F}_{k, l}^{O D C_{r a d}} \cdot \frac{\partial \tilde{F}_{k, l}^{O D C_{a n g}}}{\partial \epsilon} .
\end{aligned}
$$

The derivatives of the normalization constant are:

$$
\begin{aligned}
& \frac{\partial C^{O D C}}{\partial \rho}=\frac{1}{2 \sqrt{\frac{\sum_{k, l=1}^{N_{i m}^{s i m} \times N_{2}^{s i m}}\left(\tilde{F}_{k, l}^{O D C}\right)^{2}}{N_{1}^{s i m} N_{2}^{s i m}}}} \cdot \frac{1}{N_{1}^{s i m} N_{2}^{s i m}} \cdot \sum_{k, l=1}^{N_{1}^{s i m} \times N_{2}^{s i m}} 2 \cdot \tilde{F}_{k, l}^{O D C} \cdot \frac{\partial \tilde{F}_{k, l}^{O D C}}{\partial \rho} \\
& =\frac{\sum_{k, l=1}^{N_{1}^{s i m} \times N_{2}^{s i m}} \tilde{F}_{k, l}^{O D C} \frac{\partial \tilde{F}_{k, l}^{O D C}}{\partial \rho}}{C^{O D C} N_{1}^{s i m} N_{2}^{s i m}} \\
& \frac{\partial C^{O D C}}{\partial \delta}=\frac{\sum_{k, l=1}^{N_{1}^{s i m} \times N_{2}^{s i m}} \tilde{F}_{k, l}^{O D C} \frac{\partial \tilde{F}_{k, l}^{O D C}}{\partial \delta}}{C^{O D C} N_{1}^{s i m} N_{2}^{s i m}} \\
& \frac{\partial C^{O D C}}{\partial \theta}=\frac{\sum_{k, l=1}^{N_{1}^{s i m} \times N_{2}^{s i m}} \tilde{F}_{k, l}^{O D C} \frac{\partial \tilde{F}_{k, l}^{O D C}}{\partial \theta}}{C^{O D C} N_{1}^{s i m} N_{2}^{s i m}}
\end{aligned}
$$




$$
\frac{\partial C^{O D C}}{\partial \epsilon}=\frac{\sum_{k, l=1}^{N_{1}^{s i m} \times N_{2}^{s i m}} \tilde{F}_{k, l}^{O D C} \frac{\partial \tilde{F}_{k, l}^{O D C}}{\partial \epsilon}}{C^{O D C} N_{1}^{s i m} N_{2}^{s i m}},
$$

resulting in the following derivatives of the complete normalized filter:

$$
\begin{aligned}
& \frac{\partial F_{k, l}^{O D C}}{\partial \rho}=\frac{\frac{\partial \tilde{F}_{k, l}^{O D C}}{\partial \rho} \cdot C^{O D C}-\tilde{F}_{k, l}^{O D C} \cdot \frac{\partial C^{O D C}}{\partial \rho}}{\left(C^{O D C}\right)^{2}}, \\
& \frac{\partial F_{k, l}^{O D C}}{\partial \delta}=\frac{\frac{\partial \tilde{F}_{k, l}^{O D C}}{\partial \delta} \cdot C^{O D C}-\tilde{F}_{k, l}^{O D C} \cdot \frac{\partial C^{O D C}}{\partial \delta}}{\left(C^{O D C}\right)^{2}}, \\
& \frac{\partial F_{k, l}^{O D C}}{\partial \theta}=\frac{\frac{\partial \tilde{F}_{k, l}^{O D C}}{\partial \theta} \cdot C^{O D C}-\tilde{F}_{k, l}^{O D C} \cdot \frac{\partial C^{O D C}}{\partial \theta}}{\left(C^{O D C}\right)^{2}}, \\
& \frac{\partial F_{k, l}^{O D C}}{\partial \epsilon}=\frac{\frac{\partial \tilde{F}_{k, l}^{O D C}}{\partial \epsilon} \cdot C^{O D C}-\tilde{F}_{k, l}^{O D C} \cdot \frac{\partial C^{O D C}}{\partial \epsilon}}{\left(C^{O D C}\right)^{2}} .
\end{aligned}
$$

Derivatives of the sigmoidal non-linearity

The derivative of the standard sigmoidal function $\tilde{s}(x)$ is:

$$
\tilde{s}^{\prime}(x)=\tilde{s}(x)(1-\tilde{s}(x)) .
$$

Using this result we get the following derivatives for our sigmoidal non-linearity $s(x)$ :

$$
\begin{aligned}
\frac{\partial s(x)}{\partial x} & =\frac{\partial s(x)}{\partial \tilde{s}(x / \omega)} \cdot \frac{\partial \tilde{s}(x / \omega)}{\partial(x / \omega)} \cdot \frac{\partial(x / \omega)}{x} \\
& =\frac{2}{\omega} \tilde{s}(x / \omega)(1-\tilde{s}(x / \omega), \\
\frac{\partial s(x)}{\partial \omega} & =\frac{\partial s(x)}{\partial \tilde{s}(x / \omega)} \cdot \frac{\partial \tilde{s}(x / \omega)}{\partial(x / \omega)} \cdot \frac{\partial(x / \omega)}{\partial \omega} \\
& =\frac{-2 x}{\omega^{2}} \tilde{s}(x / \omega)(1-\tilde{s}(x / \omega)) .
\end{aligned}
$$

Derivative of the BOLD modulation transfer function

The derivative of the BOLD modulation transfer function is:

$$
\begin{aligned}
\frac{\partial M T F_{k, l}(f w h m, \beta)}{\partial f w h m} & =\frac{\partial M T F_{k, l}(f w h m, \beta)}{\partial \sigma_{B O L D}} \frac{\partial \sigma_{B O L D}}{\partial f w h m} \\
& =\frac{-4 M T F_{k, l} r(k, l)^{2} \pi^{2} f w h m}{8 \log 2}
\end{aligned}
$$

The derivative of the combined BOLD-MRI filter is:

$$
\frac{\partial F_{k, l}^{B O L D}(f w h m, \beta)}{\partial f w h m}=\frac{\partial M T F_{I_{1}^{M R I}(k), I_{2}^{M R I}(l)}(f w h m, \beta)}{\partial f w h m} \frac{N_{1}^{M R I} N_{2}^{M R I}}{N_{1}^{s i m} N_{2}^{s i m}} .
$$


Potential energy gradient components

The energy gradient is composed of the following derivatives:

$$
\begin{aligned}
\frac{\partial E(q)}{\partial n_{i, j}} & =\frac{\partial E_{G E}(q)}{\partial n_{i, j}}+\frac{\partial E_{S E}(q)}{\partial n_{i, j}}+\frac{\partial E_{\text {prior }}(q)}{\partial n_{i, j}}, \\
\frac{\partial E(q)}{\partial \rho} & =\frac{\partial E_{G E}(q)}{\partial \rho}+\frac{\partial E_{S E}(q)}{\partial \rho}+\frac{\partial E_{\text {prior }}(q)}{\partial \rho}, \\
\frac{\partial E(q)}{\partial \delta} & =\frac{\partial E_{G E}(q)}{\partial \delta}+\frac{\partial E_{S E}(q)}{\partial \delta}+\frac{\partial E_{\text {prior }}(q)}{\partial \delta}, \\
\frac{\partial E(q)}{\partial \epsilon} & =\frac{\partial E_{G E}(q)}{\partial \epsilon}+\frac{\partial E_{S E}(q)}{\partial \epsilon}+\frac{\partial E_{\text {prior }}(q)}{\partial \epsilon}, \\
\frac{\partial E(q)}{\partial \theta} & =\frac{\partial E_{G E}(q)}{\partial \theta}+\frac{\partial E_{S E}(q)}{\partial \theta}, \\
\frac{\partial E(q)}{\partial \omega} & =\frac{\partial E_{G E}(q)}{\partial \omega}+\frac{\partial E_{S E}(q)}{\partial \omega}, \\
\frac{\partial E(q)}{\partial f w h m_{G E}} & =\frac{\partial E_{G E}(q)}{\partial f w h m_{G E}}, \\
\frac{\partial E(q)}{\partial f w h m_{S E}} & =\frac{\partial E_{S E}(q)}{\partial f w h m_{S E}} .
\end{aligned}
$$

The gradient is not defind for $\left|\rho-\mu_{\rho}\right|>2 \sigma_{\rho}$ or $\delta<0$ or $\epsilon<0$ or $\omega<\omega_{\min }$ or $\omega>\omega_{\max }$ or fwhm $m_{G E}<0$ or fwhm $m_{S E}<0$ (regions were $E_{\text {prior }}(q)=\infty$ ).

Energy gradient with respect to noise variables

The derivatives of the $G E$ energy component are:

$$
\begin{aligned}
& \frac{\partial E_{G E}(q)}{\partial n_{i, j}} \\
& =\frac{1}{\hat{\sigma}_{G E}^{2}} \sum_{i^{\prime}, j^{\prime}=1}^{N_{1}^{M R I} \times N_{2}^{M R I}} d_{i^{\prime}, j^{\prime}}^{G E} \frac{\partial m r i_{i^{\prime}, j^{\prime}}}{\partial n_{i, j}} \\
& =\frac{1}{\hat{\sigma}_{G E}^{2}} \sum_{i^{\prime}, j^{\prime}=1}^{N_{1}^{M R I} \times N_{2}^{M R I}} d_{i^{\prime}, j^{\prime}}^{G E} \sum_{i^{\prime \prime}, j^{\prime \prime}=1}^{N_{1}^{s i m} \times N_{2}^{s i m}} \frac{\partial m r i_{i^{\prime}, j^{\prime}}}{\partial o d c_{i^{\prime \prime}, j^{\prime \prime}}} \frac{\partial o d c_{i^{\prime \prime}, j^{\prime \prime}}}{\partial \widetilde{o d c} c_{i^{\prime \prime}, j^{\prime \prime}}} \frac{\partial \widetilde{o d c_{i^{\prime \prime}, j^{\prime \prime}}}}{\partial n_{i, j}} \\
& =\frac{1}{\hat{\sigma}_{G E}^{2}} \sum_{i^{\prime}, j^{\prime}=1}^{N_{1}^{M R I} \times N_{2}^{M R I}} d_{i^{\prime}, j^{\prime}}^{G E} \sum_{i^{\prime \prime}, j^{\prime \prime}=1}^{N_{1}^{s i m} \times N_{2}^{s i m}} \frac{\partial m r i_{i^{\prime}, j^{\prime}}}{\partial o d c_{i^{\prime \prime}, j^{\prime \prime}}} s^{\prime}\left(\widetilde{o d c_{i^{\prime \prime}} j^{\prime \prime}}\right) \frac{\partial \widetilde{o d c_{i^{\prime \prime}, j^{\prime \prime}}}}{\partial n_{i, j}} \\
& =\frac{1}{\hat{\sigma}_{G E}^{2}} \sum_{i^{\prime \prime}, j^{\prime \prime}=1}^{N_{1}^{s i m} \times N_{2}^{s i m}} s^{\prime}\left(\widetilde{o d c}_{i^{\prime \prime}, j^{\prime \prime}}\right) \frac{\partial \widetilde{o d c_{i^{\prime \prime}, j^{\prime \prime}}}}{\partial n_{i, j}} \sum_{i^{\prime}, j^{\prime}=1}^{N_{1}^{M R I} \times N_{2}^{M R I}} d_{i^{\prime}, j^{\prime}}^{G E} \frac{\partial m r i_{i^{\prime}, j^{\prime}}}{\partial o d c_{i^{\prime \prime}, j^{\prime \prime}}}
\end{aligned}
$$

For both sums we apply D.8 (see D.2 for the definition of the zero-padding operation $\mathrm{zp}_{I_{1}^{M R I}, I_{2}^{M R I}}^{N_{1}^{s i m}, N^{s i m}}$ ):

$$
=\frac{1}{\hat{\sigma}_{G E}^{2}} \operatorname{dft} 2\left[\left(F_{k, l}^{O D C} \cdot \operatorname{idft} 2\left[\left(s^{\prime}\left(\widetilde{o d c}_{i^{\prime \prime}, j^{\prime \prime}}\right) \cdot \mathrm{zp}_{I_{1}^{M R I}, I_{2}^{M R I}}^{N_{1}^{s i m}, N_{2}^{s i m}}\left[\operatorname{dft} 2\left[\left(F_{k^{\prime}, l^{\prime}}^{M R D} \cdot \operatorname{idft} 2\left[\left(\left(d_{i^{\prime}, j^{\prime}}^{G E}\right)\right]_{k^{\prime}, l^{\prime}}\right)\right]\right]_{i^{\prime \prime}, j^{\prime \prime}}\right)\right]_{k, l}\right) .\right.\right.
$$

Similarily for the $S E$ energy component we get:

$$
\frac{\partial E_{S E}(q)}{\partial n_{i, j}}
$$




$$
=\frac{1}{\hat{\sigma}_{S E}^{2}} \operatorname{dft} 2\left[\left(F_{k, l}^{O D C} \cdot \operatorname{idft} 2\left[\left(s^{\prime}\left(\widetilde{o d c} c_{i^{\prime \prime}, j^{\prime \prime}}\right) \cdot \operatorname{zp}_{I_{1}^{M R I}, I_{2}^{M R I}}^{N_{1}^{\text {Sim }},,_{2}^{\text {Sim }}}\left[\operatorname{dft} 2\left[\left(F_{k^{\prime}, l^{\prime}}^{B O L D} \cdot \operatorname{idft} 2\left[\left(d_{i^{\prime}, j^{\prime}}^{S E}\right)\right]_{k^{\prime}, l^{\prime}}\right)\right]\right]_{i^{\prime \prime}, j^{\prime \prime}}\right)\right]_{k, l}\right)\right]
$$

The contribution of the prior energy component is:

$$
\frac{\partial E_{\text {prior }}(q)}{\partial n_{i, j}}=n_{i, j} .
$$

Energy gradient with respect to ODC filter parameters

The derivatives of the GE energy component are:

$$
\begin{aligned}
& \frac{\partial E_{G E}(q)}{\partial \rho} \\
& =\frac{1}{\sigma_{G E}^{2}} \sum_{i, j=1}^{N_{1}^{M R I} \times N_{2}^{M R I}} d_{i, j}^{G E} \frac{\partial m r i_{i, j}}{\partial \rho} \\
& =\frac{1}{\sigma_{G E}^{2}} \sum_{i, j=1}^{N_{1}^{M R I} \times N_{2}^{M R I}} d_{i, j}^{G E} \sum_{i^{\prime}, j^{\prime}=1}^{N_{1}^{s i m} \times N_{2}^{s i m}} \frac{\partial m r i_{i, j}}{\partial o d c_{i^{\prime}, j^{\prime}}} \frac{\partial o d c_{i^{\prime}, j^{\prime}}}{\partial \rho} \\
& =\frac{1}{\sigma_{G E}^{2}} \sum_{i, j=1}^{N_{1}^{M R L} \times N_{2}^{M R I}} d_{i, j}^{G E} \sum_{i^{\prime}, j^{\prime}=1}^{N_{1}^{s i m} \times N_{2}^{s i m}} \frac{\partial m r i_{i, j}}{\partial o d c_{i^{\prime}, j^{\prime}}} \frac{\partial o d c_{i^{\prime}, j^{\prime}}}{\partial \widetilde{o d c_{i^{\prime}, j^{\prime}}}} \frac{\partial \widetilde{o d c_{i^{\prime}, j^{\prime}}}}{\partial \rho} \\
& =\frac{1}{\sigma_{G E}^{2}} \sum_{i, j=1}^{N_{1}^{M R I} \times N_{2}^{M R I}} d_{i, j}^{G E} \sum_{i^{\prime}, j^{\prime}=1}^{N_{1}^{s i m} \times N_{2}^{s i m}} \frac{\partial m r i_{i, j}}{\partial o d c_{i^{\prime}, j^{\prime}}} s^{\prime}\left(\widetilde{o d c}_{i^{\prime}, j^{\prime}}\right) \frac{\partial \widetilde{o d c_{i^{\prime}, j^{\prime}}}}{\partial \rho}
\end{aligned}
$$

The last two factors together can be regarded as a pattern indexed by $i^{\prime}, j^{\prime}$. We apply D.7:

$$
=\frac{1}{\sigma_{G E}^{2}} \sum_{i, j=1}^{N_{1}^{M R I} \times N_{2}^{M R I}} d_{i, j}^{G E} \cdot \operatorname{idft} 2\left[\left(F_{k, l}^{M R I} \cdot \operatorname{dft} 2\left[\left(s^{\prime}\left(\widetilde{o d c}_{i^{\prime}, j^{\prime}}\right) \cdot \frac{\partial \widetilde{o d c}_{i^{\prime}, j^{\prime}}}{\partial \rho}\right)\right]_{\substack{k=I_{1}^{M R I}(1), \ldots, I_{M}^{M R I}\left(N_{1}^{M R I}\right), l=I_{2}^{M R I}(1), \ldots, I_{2}^{M M I}\left(N_{2}^{M R I}\right)}}\right)\right]_{i, j}
$$

$\frac{\partial \widetilde{o d c}_{c^{\prime}, j^{\prime}}}{\partial \rho}$ is the derivative of the ODC filter output $\widetilde{o d c} c_{i^{\prime}, j^{\prime}}$ with respect to a filter parameter. We apply D.9:

$$
\begin{aligned}
& =\frac{1}{\sigma_{G E}^{2}} \sum_{i, j=1}^{N_{1}^{M R I} \times N_{2}^{M R I}} d_{i, j}^{G E}
\end{aligned}
$$

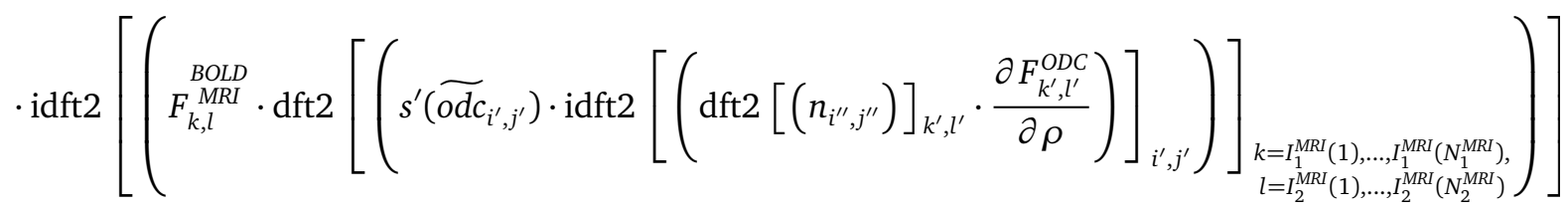

The derivatives of $E_{G E}(q)$ with respect to the remaining ODC filter parameters $\delta, \epsilon$ and $\theta$ differ only in the derivative of $F_{k^{\prime}, l^{\prime}}^{O D C}$ :

$$
\frac{\partial E_{G E}(q)}{\partial \delta}=\frac{1}{\sigma_{G E}^{2}} \sum_{i, j=1}^{N_{1}^{M R I} \times N_{2}^{M R I}} d_{i, j}^{G E}
$$




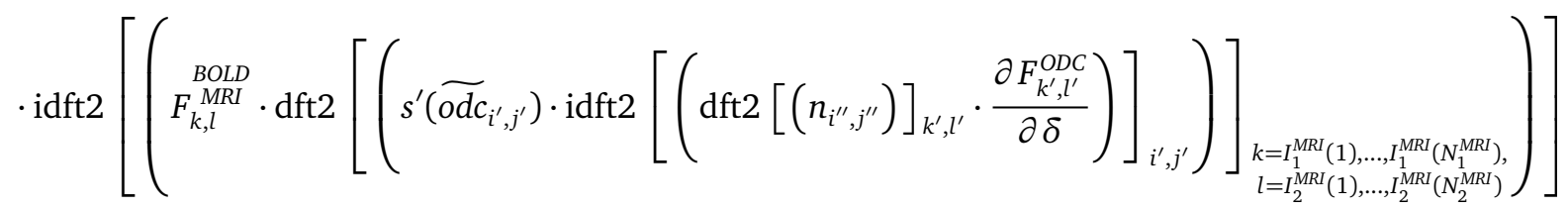

$\frac{\partial E_{G E}(q)}{\partial \epsilon}=\frac{1}{\sigma_{G E}^{2}} \sum_{i, j=1}^{N_{1}^{M R I} \times N_{2}^{M R I}} d_{i, j}^{G E}$

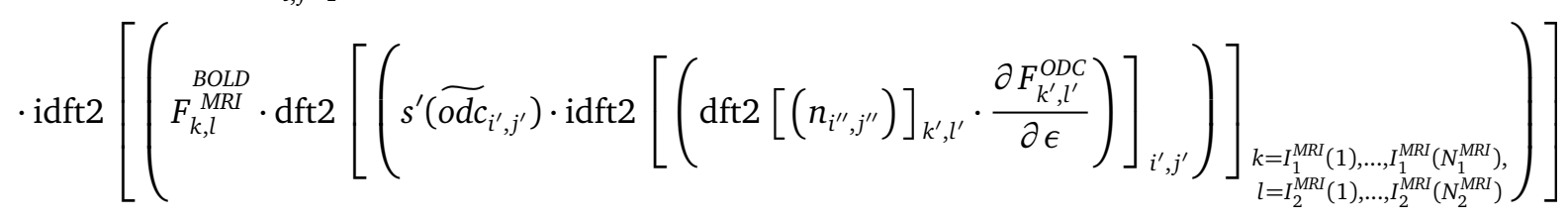

$\frac{\partial E_{G E}(q)}{\partial \theta}=\frac{1}{\sigma_{G E}^{2}} \sum_{i, j=1}^{N_{1}^{M R I} \times N_{2}^{M R I}} d_{i, j}^{G E}$

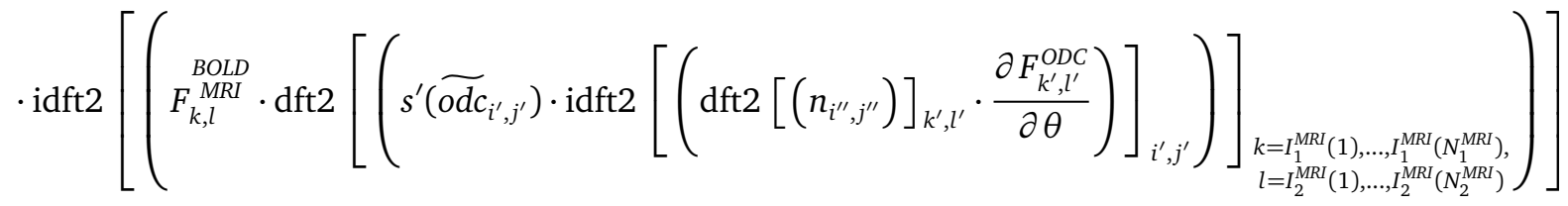

Similarily for the SE energy components we get:

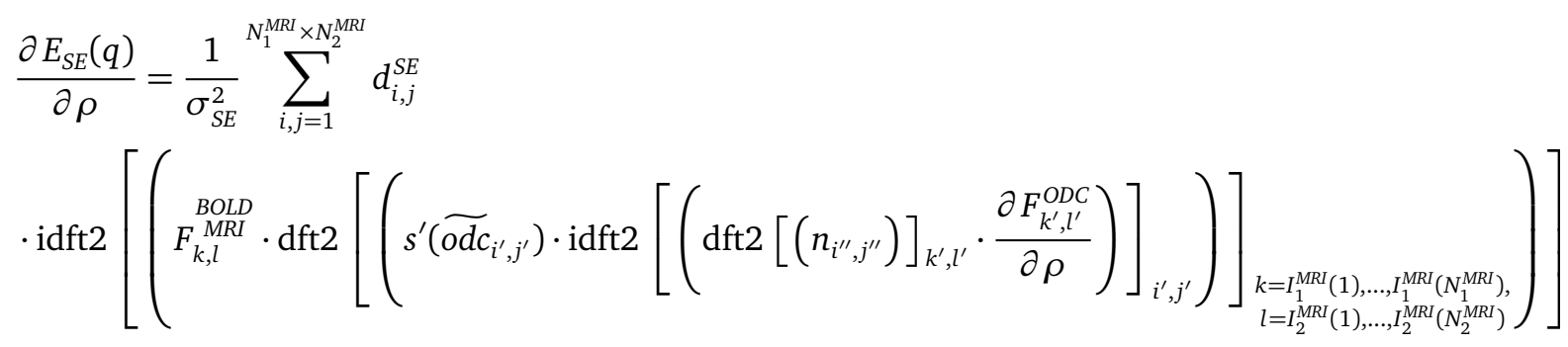

$\frac{\partial E_{S E}(q)}{\partial \delta}=\frac{1}{\sigma_{S E}^{2}} \sum_{i, j=1}^{N_{1}^{M R I} \times N_{2}^{M R I}} d_{i, j}^{S E}$

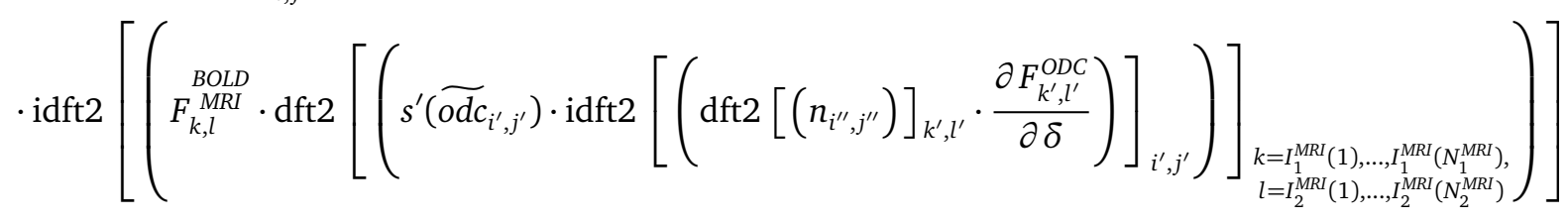

$\frac{\partial E_{S E}(q)}{\partial \epsilon}=\frac{1}{\sigma_{S E}^{2}} \sum_{i, j=1}^{N_{1}^{M R I} \times N_{2}^{M R I}} d_{i, j}^{S E}$

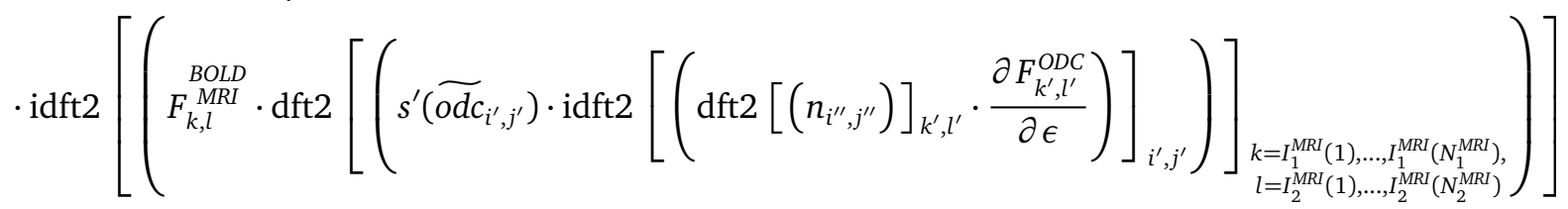




$$
\begin{aligned}
& \frac{\partial E_{S E}(q)}{\partial \theta}=\frac{1}{\sigma_{S E}^{2}} \sum_{i, j=1}^{N_{1}^{M R I} \times N_{2}^{M R I}} d_{i, j}^{S E}
\end{aligned}
$$

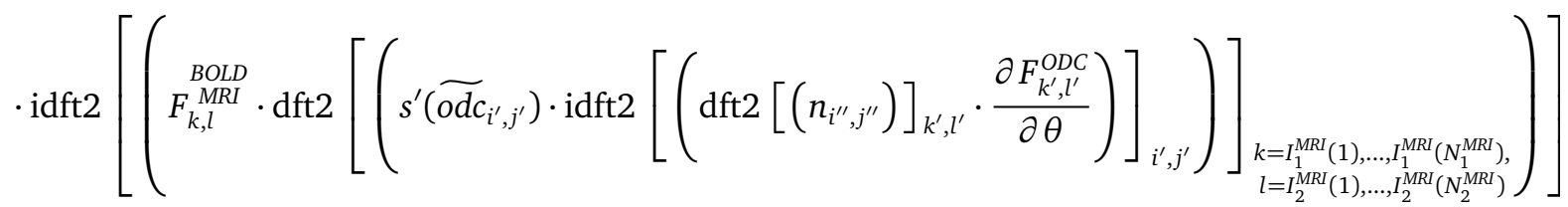

The contributions of the prior energy components are:

$$
\begin{aligned}
& \frac{\partial E_{\text {prior }}(q)}{\partial \rho}=\frac{\rho-\mu_{\rho}}{\sigma_{\rho}^{2}}, \\
& \frac{\partial E_{\text {prior }}(q)}{\partial \delta}=\frac{\delta-\mu_{\delta}}{\sigma_{\delta}^{2}}, \\
& \frac{\partial E_{\text {prior }}(q)}{\partial \epsilon}=\frac{\epsilon-\mu_{\epsilon}}{\sigma_{\epsilon}^{2}} .
\end{aligned}
$$

Energy gradient with respect to the smoothness parameter

The derivatives of the GE component are:

$$
\begin{aligned}
\frac{\partial E_{G E}(q)}{\partial \omega} & =\frac{1}{\sigma_{G E}^{2}} \sum_{i, j=1}^{N_{1}^{M R I} \times N_{2}^{M R I}} d_{i, j}^{G E} \frac{\partial m r_{m, n}^{G E}}{\partial \omega} \\
& =\frac{1}{\sigma_{G E}^{2}} \sum_{i, j=1}^{N_{1}^{M R I} \times N_{2}^{M R I}} d_{i, j}^{G E} \sum_{i^{\prime}, j^{\prime}=1}^{N_{1}^{s i m} \times N_{2}^{s i m}} \frac{\partial m r_{i, j}^{G E}}{\partial o d c_{i^{\prime}, j^{\prime}}} \frac{\partial o d c_{i^{\prime}, j^{\prime}}}{\partial \omega} \\
& =\frac{1}{\sigma_{G E}^{2}} \sum_{i, j=1}^{N_{1}^{M R I} \times N_{2}^{M R I}} d_{i, j}^{G E} \sum_{i^{\prime}, j^{\prime}=1}^{N_{1}^{s i m} \times N_{2}^{s i m}} \frac{\partial m r r_{i, j}^{G E}}{\partial o d c_{i^{\prime}, j^{\prime}}} \frac{\partial s\left(\widetilde{o d c_{i^{\prime}, j^{\prime}}}\right)}{\partial \omega}
\end{aligned}
$$

for the second sum we apply D.7:

$$
=\frac{1}{\sigma_{G E}^{2}} \sum_{i, j=1}^{N_{1}^{M R I} \times N_{2}^{M R I}} d_{i, j}^{G E} \cdot \operatorname{idft} 2\left[\left(F_{k, l}^{M R I} \cdot \operatorname{dft} 2\left[\left(\frac{\partial s\left(\widetilde{O D C}_{i^{\prime}, j^{\prime}}\right)}{\partial \omega}\right)\right]_{\substack{k=I_{1}^{M R I}(1), \ldots, I_{1}^{M R I}\left(N_{1}^{M R I}\right), l=I_{2}^{M R I}(1), \ldots, I_{2}^{M R I}\left(N_{2}^{R R I}\right)}}\right)\right]_{i, j} .
$$

Similarily for the $S E$ components we get:

$$
\frac{\partial E_{S E}(q)}{\partial \omega}=\frac{1}{\sigma_{S E}^{2}} \sum_{i, j=1}^{N_{1}^{M R I} \times N_{2}^{M R I}} d_{i, j}^{S E} \cdot \operatorname{idft} 2\left[\left(F_{k, l}^{B O L D} \cdot \operatorname{dft} 2\left[\left(\frac{\partial s\left(\widetilde{O D C}_{i^{\prime}, j^{\prime}}\right)}{\partial \omega}\right)\right]_{\substack{k=I_{M}^{M R I}(1), \ldots, I_{1}^{M R I}\left(N_{M}^{M R I}\right), l=I_{2}^{M R I}(1), \ldots, I_{2}^{M R I}\left(N_{2}^{M R I}\right)}}\right)\right]_{i, j} .
$$


Energy gradient with respect to BOLD PSF width

The derivatives of the GE component are:

$$
\frac{\partial E_{G E}(q)}{\partial f w h m_{G E}}=\frac{1}{\sigma_{G E}^{2}} \sum_{i, j=1}^{N_{1}^{M R I} \times N_{2}^{M R I}} d_{i, j}^{G E} \frac{\partial m r i_{i, j}^{G E}}{\partial f w h m_{G E}}
$$

We apply D.9:

$$
=\frac{1}{\sigma_{G E}^{2}} \sum_{i, j=1}^{N_{1}^{M R I} \times N_{2}^{M R I}} d_{i, j}^{G E} \cdot \operatorname{idft2}\left[\left(\operatorname{dft} 2\left[\left(o d c_{i^{\prime}, j^{\prime}}\right)\right]_{\substack{k=I_{1}^{M R I}(1), \ldots, I_{1}^{M R I}\left(N_{1}^{M R I}\right), l=I_{2}^{M R I}(1), \ldots, I_{2}^{M R I}\left(N_{2}^{M R I}\right)}} \cdot \frac{\partial F_{k, l}^{B O L D}}{\partial f w h m_{G E}}\right)\right]_{i, j} .
$$

Similarily for the $S E$ components we get:

$$
\frac{\partial E_{S E}(q)}{\partial f w h m_{S E}}=\frac{1}{\sigma_{S E}^{2}} \sum_{i, j=1}^{N_{1}^{M R I} \times N_{2}^{M R I}} d_{i, j}^{S E} \cdot \operatorname{idft2}\left[\left(\operatorname{dft} 2\left[\left(\operatorname{odc}_{i^{\prime}, j^{\prime}}\right)\right]_{\substack{k=I_{1}^{M R I}(1), \ldots, I_{1}^{M R I}\left(N_{1}^{M R I}\right), l=I_{2}^{M R I}(1), \ldots, I_{2}^{M R I}\left(N_{2}^{M R I}\right)}} \cdot \frac{\partial F_{k, l}^{B O L D}}{\partial f w h m_{S E}}\right)\right]_{i, j} .
$$

\section{Appendix D. Efficient computation of some expressions consisting of derivatives of filtering op- erations}

Let us assume we have a pattern $\left(x_{i, j}\right)$ of size $N_{1}^{i n} \times N_{2}^{i n}$ (the input), whose discrete Fourier transform is $\left(X_{k, l}\right)$. We filter (or equivalently: convolve) that pattern using a linear filter $\left(F_{k, l}\right)$ defined in frequency space and of size $N_{1}^{\text {out }} \times N_{2}^{\text {out }}$. The filtering is carried out as:

$$
Y_{k, l}=F_{k, l} \cdot X_{I_{1}(k), I_{2}(l)},
$$

where $\left(Y_{k, l}\right)$ is the discrete Fourier transform of the filtered, $N_{1}^{\text {out }} \times N_{2}^{\text {out }} \operatorname{sized~pattern~}\left(y_{m, n}\right)$ (the output). $I_{1}$ and $I_{2}$ are index functions that allow to assign a specific subset of elements of $\left(X_{k, l}\right)$ to $\left(Y_{k, l}\right)$ (e.g. when restricting the spatial frequency space representation in order to simulate MRI sampling). Note that for $N_{1}^{\text {out }}=N_{1}^{\text {in }}, N_{2}^{\text {out }}=N_{2}^{\text {in }}, I_{1}(k)=k$ and $I_{2}(l)=l$ this formalism describes a normal filtering operation in which the size and structure of the spatial frequency space is not altered.

Our goal here is to derive alternate formulations for some derivative expressions that result in a more efficient computation of gradients for models that contain such filtering operations.

\section{Definitions}

We define a zero-padding operation $\mathrm{zp}_{I_{1}, I_{2}}^{N_{1}^{\text {in }}, N_{2}^{\text {in }}}$ that allows us to up-sample patterns with size equal to the output pattern to match the size of the input pattern:

$$
\left(\bar{Y}_{k, l}\right)=\operatorname{zp}_{I_{1}, I_{2}}^{N_{1}^{\text {in }}, N_{2}^{i n}}\left[\left(Y_{k, l}\right)\right],
$$

where $\left(\bar{Y}_{k, l}\right)$ is of size $N_{1}^{i n} \times N_{2}^{i n}$ such that:

$$
\bar{Y}_{k, l}=\left\{\begin{array}{ll}
Y_{k^{\prime}, l^{\prime}} & \text { if there exist }\left(k^{\prime}, l^{\prime}\right) \text { with } I_{1}\left(k^{\prime}\right)=k \text { and } I_{2}\left(l^{\prime}\right)=l \\
0 & \text { otherwise }
\end{array} .\right.
$$

We use the following definitions of the two-dimensional discrete Fourier transform $\left(X_{k, l}\right)=\operatorname{dft} 2\left[\left(x_{i}\right.\right.$, and its inverse $\left(x_{i, j}\right)=\operatorname{idft} 2\left[\left(X_{k, l}\right)\right]$, where $\left(x_{i, j}\right)$ and $\left(X_{k, l}\right)$ are of size $N_{1} \times N_{2}$ :

$$
X_{k, l}=\sum_{i, j=1}^{N_{1} \times N_{2}} x_{i, j} e^{-\dot{\mathrm{i} 2} \pi \frac{(i-1)(k-1)}{N_{1}^{i n}}} e^{-\dot{\mathrm{i}} 2 \pi \frac{(j-1)(l-1)}{N_{2}^{i n}}}
$$




$$
x_{i, j}=\frac{1}{N_{1} N_{2}} \sum_{k, l=1}^{N_{1}^{i n} \times N_{2}^{i n}} X_{k, l} e^{\dot{\mathrm{i}} 2 \pi \frac{(k-1)(i-1)}{N_{1}^{i n}}} e^{\dot{\mathrm{i}} 2 \pi \frac{(l-1)(j-1)}{N_{2}^{i n}}},
$$

with partial derivatives given as:

$$
\begin{aligned}
& \frac{\partial X_{k, l}}{\partial x_{i, j}}=e^{-\dot{\mathrm{i}} 2 \pi \frac{(i-1)(k-1)}{N_{1}^{i n}}} e^{-\dot{\mathrm{i}} 2 \pi \frac{(j-1)(l-1)}{N_{2}^{i n}}}, \\
& \frac{\partial x_{i, j}}{\partial X_{k, l}}=\frac{1}{N_{1}^{i n} N_{2}^{i n}} e^{\dot{\mathrm{i}} 2 \pi \frac{(k-1)(i-1)}{N_{1}^{i n}}} e^{\mathrm{i} 2 \pi \frac{(l-1)(j-1)}{N_{2}^{i n}}} .
\end{aligned}
$$

Weighted sums of derivatives with respect to input

The first expression of interest is the weighted sum (weights given as $\left(g_{i, j}\right)$ ) of the derivatives of an arbitrary output element $y_{i, j}$ with respect to all input elements $x_{i^{\prime}, j^{\prime}}$. We derive a formula that allows to compute this expressions for all output elements $y_{i, j}$ simultaneously using one dft2 and one idft2 operation.

$$
\begin{aligned}
& \sum_{i^{\prime}, j^{\prime}=1}^{N_{1}^{i n} \times N_{2}^{\text {in }}} g_{i^{\prime}, j^{\prime}} \frac{\partial y_{i, j}}{\partial x_{i^{\prime}, j^{\prime}}} \\
& =\sum_{i^{\prime}, j^{\prime}=1}^{N_{1}^{\text {in }} \times N_{2}^{\text {in }}} g_{i^{\prime}, j^{\prime}} \sum_{k, l=1}^{N_{1}^{\text {out }} \times N_{2}^{\text {out }}} \frac{\partial y_{i, j}}{\partial Y_{k, l}} \sum_{k^{\prime}, l^{\prime}=1}^{N_{1}^{\text {in }} \times N_{2}^{i n}} \frac{\partial Y_{k, l}}{\partial X_{k^{\prime}, l^{\prime}}} \frac{\partial X_{k^{\prime}, l^{\prime}}}{\partial x_{i^{\prime}, j^{\prime}}} .
\end{aligned}
$$

Because of D.1, the second to last term is equal to $F_{k, l}$, if $\left(k^{\prime}, l^{\prime}\right)=\left(I_{1}(k), I_{2}(l)\right)$ and 0 otherwise:

$$
=\sum_{i^{\prime}, j^{\prime}=1}^{N_{1}^{\text {in }} \times N_{2}^{\text {in }}} g_{i^{\prime}, j^{\prime}} \sum_{k, l=1}^{N_{1}^{\text {out }} \times N_{2}^{\text {out }}} \frac{\partial y_{i, j}}{\partial Y_{k, l}} F_{k, l} \frac{\partial X_{I_{1}(k), I_{2}(l)}}{\partial x_{i^{\prime}, j^{\prime}}}
$$

Furthermore making use of D.5 and D.6 we get:

$$
\begin{aligned}
& =\sum_{i^{\prime}, j^{\prime}=1}^{N_{1}^{\text {in }} \times N_{2}^{\text {in }}} g_{i^{\prime}, j^{\prime}} \frac{1}{N_{1}^{\text {out }} N_{2}^{\text {out }}} \sum_{k, l=1}^{N_{1}^{\text {out }} \times N_{2}^{\text {out }}} e^{\dot{\mathrm{i}} 2 \pi \frac{(k-1)(i-1)}{N_{1}^{\text {out }}}} e^{\dot{\mathrm{i}} 2 \pi \frac{(l-1)(j-1)}{N_{2}^{\text {out }}}} e^{-\dot{\mathrm{i}} 2 \pi \frac{\left(i^{\prime}-1\right)\left(I_{1}(k)-1\right)}{N_{1}^{\text {in }}}} e^{-\dot{\mathrm{i} 2} 2 \pi \frac{\left(j^{\prime}-1\right)\left(I_{2}(l)-1\right)}{N_{2}^{\text {in }}}} \\
& =\frac{1}{N_{1}^{\text {out }} N_{2}^{\text {out }}} \sum_{k, l=1}^{N_{1}^{\text {out }} \times N_{2}^{\text {out }}} e^{\dot{\mathrm{i}} 2 \pi \frac{(k-1)(i-1)}{N_{1}^{\text {out }}}} e^{\dot{\mathrm{i}} 2 \pi \frac{(l-1)(j-1)}{N_{2}^{\text {out }}}} \sum_{i^{\prime}, j^{\prime}=1}^{N_{1}^{\text {in }} \times N_{2}^{\text {in }}} g_{i^{\prime}, j^{\prime}} e^{-\dot{\mathrm{i}} 2 \pi \frac{\left(i^{\prime}-1\right)\left(I_{1}(k)-1\right)}{N_{1}^{\text {in }}}} e^{-\dot{\mathrm{i}} 2 \pi \frac{\left(j^{\prime}-1\right)\left(I_{2}(l)-1\right)}{N_{2}^{\text {in }}}} \\
& =\frac{1}{N_{1}^{\text {out }} N_{2}^{\text {out }}} \sum_{k, l=1}^{N_{1}^{\text {out }} \times N_{2}^{\text {out }}} F_{k, l} e^{\mathrm{i} 2 \pi \frac{(k-1)(i-1)}{N_{1}^{\text {out }}}} e^{\dot{\mathrm{i}} 2 \pi \frac{(l-1)(j-1)}{N_{2}^{\text {out }}}} \mathrm{dft} 2\left[\left(g_{i^{\prime}, j^{\prime}}\right)\right]_{I_{1}(k), I_{2}(l)} \\
& =\operatorname{idft} 2\left[\left(F_{k, l} \cdot \mathrm{dft} 2\left[\left(g_{i^{\prime}, j^{\prime}}\right)\right]_{I_{1}(k), I_{2}(l)}\right)\right]_{i, j} \cdot
\end{aligned}
$$

A related expression is the weighted sum of the derivatives of all output elements $y_{i^{\prime}, j^{\prime}}$ with respect to an arbitrary but specific input element $x_{i, j}$. Again, we derive a formula that allows to compute this expressions for all input elements $x_{i, j}$ simultaneously using one dft2 and one idft2 operation.

$$
\sum_{i^{\prime}, j^{\prime}=1}^{N_{1}^{o u t} \times N_{2}^{\text {out }}} g_{i^{\prime}, j^{\prime}} \frac{\partial y_{i^{\prime}, j^{\prime}}}{\partial x_{i, j}}
$$




$$
=\sum_{i^{\prime}, j^{\prime}=1}^{N_{1}^{\text {out }} \times N_{2}^{\text {out }}} g_{i^{\prime}, j^{\prime}} \sum_{k, l=1}^{N_{1}^{\text {out }} \times N_{2}^{\text {out }}} \frac{\partial y_{i^{\prime}, j^{\prime}}}{\partial Y_{k, l}} \sum_{k^{\prime}, l^{\prime}=1}^{N_{1}^{\text {in }} \times N_{2}^{\text {in }}} \frac{\partial Y_{k, l}}{\partial X_{k^{\prime}, l^{\prime}}} \frac{\partial X_{k^{\prime}, l^{\prime}}}{\partial x_{i, j}}
$$

Because of D.1, the second to last term is equal to $F_{k, l}$, if $\left(k^{\prime}, l^{\prime}\right)=\left(I_{1}(k), I_{2}(l)\right)$ and 0 otherwise:

$$
=\sum_{i^{\prime}, j^{\prime}=1}^{N_{1}^{\text {out }} \times N_{2}^{\text {out }}} g_{i^{\prime}, j^{\prime}} \sum_{k, l=1}^{N_{1}^{\text {out }} \times N_{2}^{\text {out }}} \frac{\partial y_{i^{\prime}, j^{\prime}}}{\partial Y_{k, l}} F_{k, l} \frac{\partial X_{I_{1}(k), I_{2}(l)}}{\partial x_{i, j}} .
$$

Furthermore making use of D.5 and D.6 we get:

$$
\begin{aligned}
& =\sum_{i^{\prime}, j^{\prime}=1}^{N_{1}^{\text {out }} \times N_{2}^{\text {out }}} g_{i^{\prime}, j^{\prime}} \frac{1}{N_{1}^{\text {out }} N_{2}^{\text {out }}} \sum_{k, l=1}^{N_{1}^{\text {out }} \times N_{2}^{\text {out }}} F_{k, l} e^{\dot{\mathrm{i} 2} 2 \frac{(k-1)\left(i^{\prime}-1\right)}{N_{1}^{\text {out }}}} e^{\mathrm{i} 2 \pi \frac{(l-1)\left(j^{\prime}-1\right)}{N_{2}^{\text {out }}}} e^{-\dot{\mathrm{i}} 2 \pi \frac{(i-1)\left(I_{1}(k)-1\right)}{N_{1}^{i n}}} e^{-\dot{\mathrm{i} 2} 2 \pi \frac{(j-1)\left(I_{2}(l)-1\right)}{N_{2}^{\text {in }}}} \\
& =\frac{1}{N_{1}^{\text {out }} N_{2}^{\text {out }}} \sum_{k, l=1}^{N_{1}^{\text {out }} \times N_{2}^{\text {out }}} F_{k, l} e^{-\dot{\mathrm{i} 2} 2 \frac{(i-1)\left(I_{1}(k)-1\right)}{N_{1}^{\text {in }}}} e^{-\dot{\mathrm{i} 2} \pi \frac{(j-1)\left(I_{2}(l)-1\right)}{N_{2}^{\text {in }}}} \sum_{i^{\prime}, j^{\prime}=1}^{N_{1}^{\text {out }} \times N_{2}^{\text {out }}} g_{i^{\prime}, j^{\prime}} e^{\dot{\mathrm{i} 2 \pi \frac{(k-1)\left(i^{\prime}-1\right)}{N_{1}^{\text {out }}}} e^{\mathrm{i} 2 \pi \frac{(l-1)\left(j^{\prime}-1\right)}{N_{2}^{\text {out }}}}}
\end{aligned}
$$

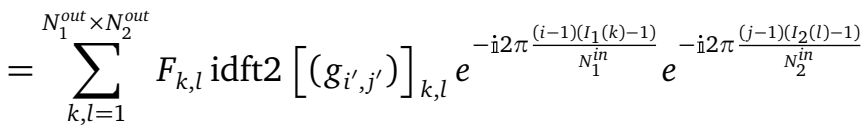

Instead of summing over the output indices $k, l=1, \ldots, N_{1}^{\text {out }} \times N_{2}^{\text {out }}$, we can zero-pad $F_{k, l} \operatorname{idft} 2\left[\left(g_{i^{\prime}, j^{\prime}}\right)\right]_{k, l}$ and sum over the input indices $k^{\prime}, l^{\prime}=1, \ldots, N_{1}^{i n} \times N_{2}^{i n}$. The additional elements are 0 due to the zeropadding and will not contribute to the sum.

$$
\begin{aligned}
& =\sum_{k^{\prime}, l^{\prime}=1}^{N_{1}^{i n} \times N_{2}^{i n}} \operatorname{zp}_{I_{1}, I_{2}}^{N_{1}^{i n}, N_{2}^{i n}}\left[\left(F_{k, l} \cdot \operatorname{idft} 2\left[\left(g_{i^{\prime}, j^{\prime}}\right)\right]_{k, l}\right)\right]_{k^{\prime}, l^{\prime}} e^{-\dot{\mathrm{i} 2} 2 \frac{(i-1)\left(k^{\prime}-1\right)}{N_{1}^{i n}}} e^{-\dot{\mathrm{i} 2} 2 \pi \frac{\left.(j-1) l^{\prime}-1\right)}{N_{2}^{i n}}} \\
& =\operatorname{dft} 2\left[\operatorname{zp}_{I_{1}, I_{2}}^{N_{1}^{i n}, N_{2}^{i n}}\left[\left(F_{k, l} \cdot \operatorname{idft} 2\left[\left(g_{i^{\prime}, j^{\prime}}\right)\right]_{k, l}\right)\right]\right]_{i, j} \cdot
\end{aligned}
$$

Derivative with respect to a filter parameter

Let us assume that the filter representation $\left(F_{k, l}\right)$ depends on some parameter $q$. We derive a formula that allows to calculate the partial derivatives of all output elements $y_{m, n}$ with respect to $q$ simultaneously using one $\mathrm{dft} 2$ and one idft 2 operation.

$$
\frac{\partial y_{i, j}}{\partial q}=\sum_{k^{\prime}, l^{\prime}}^{N_{1}^{\text {out }} \times N_{2}^{\text {out }}} \frac{\partial y_{i, j}}{\partial Y_{k^{\prime}, l^{\prime}}} \sum_{k^{\prime \prime}, l^{\prime \prime}}^{N_{1}^{\text {out }} \times N_{2}^{\text {out }}} \frac{\partial Y_{k^{\prime}, l^{\prime}}}{\partial F_{k^{\prime \prime}, l^{\prime \prime}}} \frac{\partial F_{k^{\prime \prime}, l^{\prime \prime}}}{\partial q}
$$

Because of D.1, the second to last term is equal to $X_{I_{1}\left(k^{\prime}\right), I_{2}\left(l^{\prime}\right)}$ if $\left(k^{\prime \prime}, l^{\prime \prime}\right)=\left(I_{1}\left(k^{\prime}\right), I_{2}\left(l^{\prime}\right)\right)$ and 0 otherwise:

$$
=\sum_{k^{\prime}, l^{\prime}}^{N_{1}^{\text {out }} \times N_{2}^{\text {out }}} \frac{\partial y_{i, j}}{\partial Y_{k^{\prime}, l^{\prime}}} X_{I_{1}\left(k^{\prime}\right), I_{2}\left(l^{\prime}\right)} \frac{\partial F_{k^{\prime}, l^{\prime}}}{\partial q}
$$

We apply D.6:

$$
\begin{aligned}
& =\sum_{k^{\prime}, l^{\prime}}^{N_{1}^{\text {out }} \times N_{2}^{\text {out }}} \frac{1}{N_{1}^{\text {out }} N_{2}^{\text {out }}} e^{\mathrm{i} 2 \pi \frac{\left(k^{\prime}-1\right)(i-1)}{N_{1}^{\text {out }}}} e^{\dot{\mathrm{i} 2} 2 \pi \frac{\left(l^{\prime}-1\right)(j-1)}{N_{2}^{\text {out }}}} X_{I_{1}\left(k^{\prime}\right), I_{2}\left(l^{\prime}\right)} \frac{\partial F_{k^{\prime}, l^{\prime}}}{\partial q} \\
& =\operatorname{idft} 2\left[\left(\operatorname{dft} 2\left[\left(x_{i^{\prime}, j^{\prime}}\right)\right]_{I_{1}\left(k^{\prime}\right), I_{2}\left(l^{\prime}\right)} \cdot \frac{\partial F_{k^{\prime}, l^{\prime}}}{\partial q}\right)\right]_{i, j} \cdot
\end{aligned}
$$




\section{Supplementary Material}

GE

A

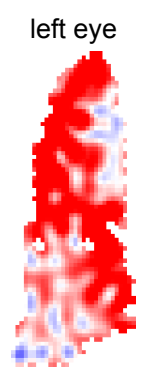

B

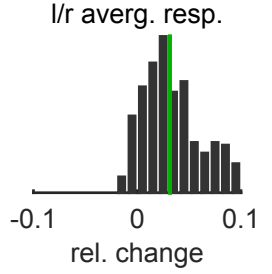

C

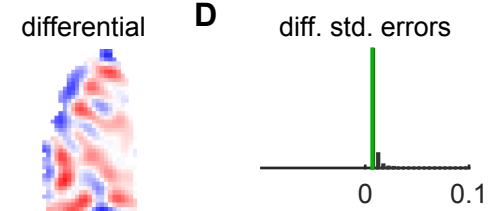

rel. change
SE

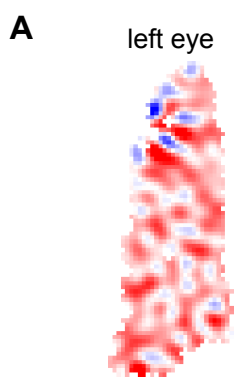

C

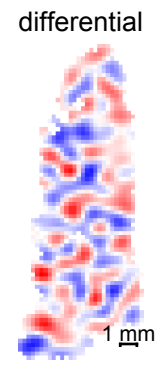

B

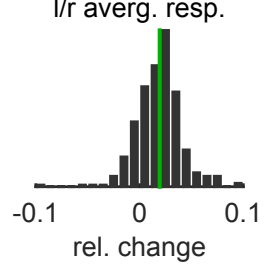

right eye

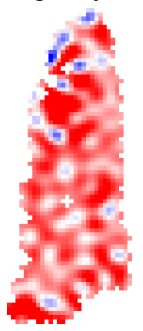

D

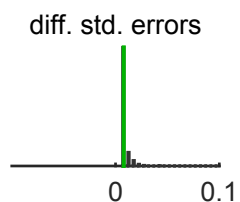

rel. change

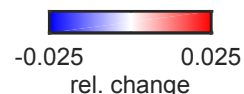

Sup. Fig. 1 fMRI ODC data. Results from the GLM analysis of fMRI data from subject 2 for GE (left) and SE (right). A Responses to left and right eye stimulation relative to baseline. B The response maps to the left and right eyes from A were averaged. B shows the distribution of the average response. Its median (in green) was used to set the overall amplitude of the BOLD response model. C The difference between left and right eye responses yields the differential ODC map. D The distribution of standard errors of all differential responses. From this distribution we estimated the noise level used by the model. The color look-up-table applies to all response maps. 
bioRxiv preprint doi: https://doi.org/10.1101/097287; this version posted December 29, 2016. The copyright holder for this preprint (which was not certified by peer review) is the author/funder, who has granted bioRxiv a license to display the preprint in perpetuity. It is made available under aCC-BY-NC-ND 4.0 International license.

\section{A Parameters}
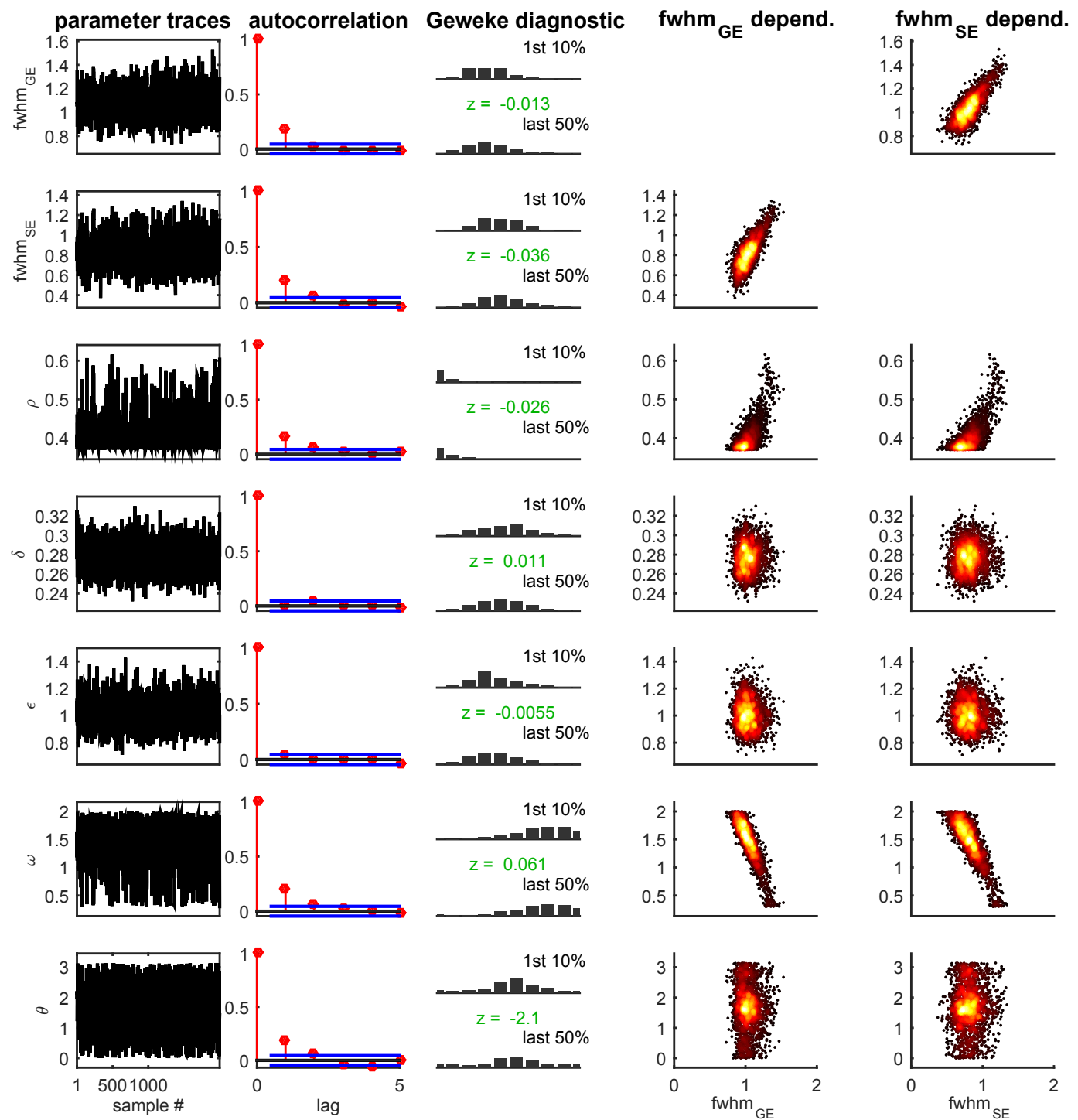

\section{B Noise values}
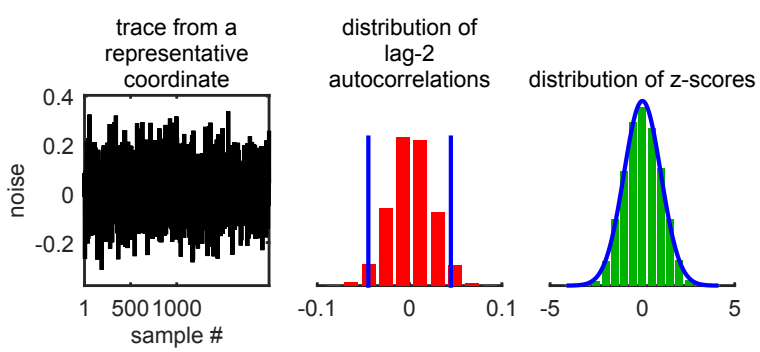

Sup. Fig. 2 Convergence diagnostics of Markov Chain Monte Carlo sampling. Markov Chain Monte Carlo needs to run for a sufficient number of iterations in order to yield samples from the modeled probability distribution. Indications for convergence are: (1) stationarity of the parameter sampling distributions, and (2) sample autocorrelations decrease rapidly with increasing lag, relative to the total number of samples. This figure examines convergence for subject 2. The upper part of the figure (A) shows diagnostics for the standard model parameters. The bottom part (B) shows diagnostics for the white noise values that act as parameters to determine the ODC pattern. The first column (A and B) shows traces of the sampled parameters. For the noise values (B), one exemplary trace is shown from the center of the map. The second column (A and B) shows sample autocorrelations as a function of lag. The horizontal blue lines (A) indicate the 95\%-confidence bounds around 0 for a white noise process. Consecutive samples 
bioRxiv preprint doi: https://doi.org/10.1101/097287; this version posted December 29, 2016 . The copyright holder for this preprint (which was not certified by peer review) is the author/funder, who has granted bioRxiv a license to display the preprint in perpetuity. It is made available under aCC-BY-NC-ND 4.0 International license.

$(\mathrm{lag}=1)$ show low autocorrelation. However, samples of lag 2 (and higher) show autocorrelation estimates that are comparable to those obtained from uncorrelated white noise. In $\mathrm{B}$, the autocorrelation from the noise samples are summarized by the histogram of lag- 2 autocorrelations from all coordinates together. Here, 95\%-confidence bounds for a white noise process are indicated by vertical blue lines. The third column (A and B) presents the Geweke convergence (stationarity) diagnostic, which is a z-test (zscores shown in green) for testing whether the means of the first $10 \%$ and last $50 \%$ of samples are different. In A, 2 histograms per each parameter show how similar their respective distributions are. In $\mathrm{B}$, the z-scores from the noise samples are shown as a histogram together with a blue plot of the standard normal probability density representing the null-hypothesis of $\mathrm{z}=0$. The last two columns (A) show the sample covariation between each parameter (vertical axis) and the GE and SE point-spread function FWHM (horizontal axis). 